\title{
Discrete-time phytoplankton-zooplankton model with bifurcations and chaos
}

\author{
A.Q. $\operatorname{Khan}^{1 *}$ (D) and M.B. Javaid
}

"Correspondence:

abdulqadeerkhan1@gmail.com

'Department of Mathematics, University of Azad Jammu and Kashmir, Muzaffarabad 13100, Pakistan

\section{Springer}

\begin{abstract}
The local dynamics with different topological classifications, bifurcation analysis, and chaos control for the phytoplankton-zooplankton model, which is a discrete analogue of the continuous-time model by a forward Euler scheme, are investigated. It is proved that the discrete-time phytoplankton-zooplankton model has trivial and semitrivial fixed points for all involved parameters, but it has an interior fixed point under the definite parametric condition. Then, by linear stability theory, local dynamics with different topological classifications are investigated around trivial, semitrivial, and interior fixed points. Further, for the discrete-time phytoplankton-zooplankton model, the existence of periodic points is also investigated. The existence of possible bifurcations around trivial, semitrivial, and interior fixed points is also investigated, and it is proved that there exists a transcritical bifurcation around a trivial fixed point. It is also proved that around trivial and semitrivial fixed points of the phytoplankton-zooplankton model there exists no flip bifurcation, but around an interior fixed point there exist both Neimark-Sacker and flip bifurcations. From the viewpoint of biology, the occurrence of Neimark-Sacker implies that there exist periodic or quasi-periodic oscillations between phytoplankton and zooplankton populations. Next, the feedback control method is utilized to stabilize chaos existing in the phytoplankton-zooplankton model. Finally, simulations are presented to validate not only obtained results but also the complex dynamics with orbits of period-8, 9, 10,11, 14, 15 and chaotic behavior of the discrete-time phytoplankton-zooplankton model.
\end{abstract}

MSC: 70K50; 92D25; 40A05

Keywords: Phytoplankton-zooplankton model; Bifurcations; Numerical simulation; Chaos

\section{Introduction}

The rapid population and equally rapid decline are features of phytoplankton. Phytoplankton are temperature and nutrients sensitive. Their growth rate and reduction depend upon several features like temperature, nutrients, season, and the place of occurrence. Phytoplankton is commonly found in two types: spring blooms and red tides. As the name shows, spring blooms outbreak is seasonal and depends upon availability of nutrients. They are more sensitive as compared to other phytoplankton. Their outbreaks and survival are shorter than those of others. The name red tides is derived from their appearance.

(c) The Author(s) 2021. This article is licensed under a Creative Commons Attribution 4.0 International License, which permits use sharing, adaptation, distribution and reproduction in any medium or format, as long as you give appropriate credit to the original author(s) and the source, provide a link to the Creative Commons licence, and indicate if changes were made. The images or other third party material in this article are included in the article's Creative Commons licence, unless indicated otherwise in a credit line to the material. If material is not included in the article's Creative Commons licence and your intended use is not permitted by statutory regulation or exceeds the permitted use, you will need to obtain permission directly from the copyright holder. To view a copy of this licence, visit http://creativecommons.org/licenses/by/4.0/. 
The red tides are more usual and formal in coastal areas strong enough to absorb heat and toxic nutrients to survive. Red tides survive for several months, and their blooming is for some weeks or months. The evaluation in red tide species helps them to survive in the environment. The phytoplankton is divided into two categories: the first one shows rapid growth, while the second shows low rate of growth. The growth rate of these species depends upon environmental conditions and availability of nutrients. Some species of phytoplankton secrete a large amount of toxins that is the cause of fishes. When a bloom of a specific destructive phytoplankton occurs, the aggregate impact of all the poison released may influence other life forms, causing mass mortality. Such extraordinary concentrations or blooms are responsible for the enormous localized mortality watched in fishes and invertebrates in different places [1]. There has been a worldwide increment in harmful plankton blossoms in the final three decades, see the cited bibliography therein for more consultation on this topic [2-5], and considerable logical consideration towards harmful algal sprouts has been paid in recent years $[6,7]$.

Hereafter before giving the mathematical modeling of the phytoplankton-zooplankton model, first we give some characteristics of red tides. It is pointed out in [1] that a phytoplankton-zooplankton model describes the detail of any particular species such as location, reproduction, and the following other features of different species of red tides.

- On the basis of existence, red tides are classified into two classes. The population of the first one remains constant throughout seasons of several months and that of the second does not remain constant.

- The evolutionary process in that species is very slow, which helps to control the environmental conditions preventing from the predators and the effects of toxins.

- The growth rate of red tides depends upon different environmental factors such as temperature, nutrients, trace elements, and pollution. This mechanism also describes the survival of that species in a changing environment. The population of that species increases or decreases due to environmental factors.

- The occurrence of that species in a season is cyclic in nature: after getting maturity it returns to the original forum. This model also describes the mechanism of their rapid growth and recycling in the environment.

It is also pointed out in [1] that a phytoplankton-zooplankton model explains the red tide environment as a constant system with population emerging with time, exemplified by ordinary differential equations. There are many expedient nominees for the role of refractory variable, but these are arranged into two classes: intrinsic and extrinsic. Within the mathematical model each complement would be denoted by a pair of coupled ordinary differential equations. Many accessible refractory variables are neither entirely intrinsic nor extrinsic. A similar situation would apparently hold for iron concentrations. The attribute of the elicit mechanism ascertained in the model lies in the interaction of growth rate of phytoplankton with the grazing rate of zooplankton. The mechanism is then modeled in the phytoplankton evolution as follows:

$$
\frac{d P}{d t}=r P\left(1-\frac{P}{K}\right)-R_{m} Z \frac{P^{2}}{\alpha^{2}+P^{2}}
$$

where $P$ and $Z$ respectively denote the populations of phytoplankton and zooplankton. Moreover, the parameter $r$ denotes the gross rate of production of phytoplankton, $R_{m}$ is the maximum specific predation rate, $K$ is carrying capacity, and $\alpha$ governs how quickly that 
maximum is attained as prey densities increase. It is specified in [1] that Holling type-III utilized in several biological models and most commonly Holling type-III shape are as the Michaelis-Menten grazing function $R_{m} Z \frac{P}{\alpha+P}$ and the Ivlev grazing function $R_{m} Z\left(1-e^{-\lambda P}\right)$. Advance headstrong calculated within the system is the populace of zooplankton. In this manner, the rate of generation of zooplankton is controlled by the population thickness of phytoplankton, whereas their misfortune from the framework is through passing and natural predation by higher individuals of the nourishment web. So the full phytoplanktonzooplankton system takes the following form [1]:

$$
\frac{d P}{d t}=r P\left(1-\frac{P}{K}\right)-R_{m} Z \frac{P^{2}}{\alpha^{2}+P^{2}}, \quad \frac{d Z}{d t}=\gamma Z R_{m} \frac{P^{2}}{\alpha^{2}+P^{2}}-\mu Z .
$$

It is noted that in (2), parameter $\mu$ is the specific rate of elimination of zooplankton by passing out, and predacity is modeled as being relative to zooplankton population and $\gamma$ is the ratio of biomass consumed to biomass of new herbivores produced. Now model (2) takes the following form:

$$
R_{m} \frac{d \widetilde{P}}{d \widetilde{t}}=r \widetilde{P}(1-\widetilde{P})-R_{m} \widetilde{Z} \frac{K^{2} \widetilde{P}^{2}}{\alpha^{2}+K^{2} \widetilde{P}^{2}}, \quad R_{m} \frac{d \widetilde{Z}}{d \widetilde{t}}=\gamma R_{m} \widetilde{Z} \frac{K^{2} \widetilde{P}^{2}}{\alpha^{2}+K^{2} \widetilde{P}^{2}}-\mu \widetilde{Z}
$$

by using the following transformations:

$$
P=K \widetilde{P}, \quad Z=K \widetilde{Z}, \quad t=\frac{\widetilde{t}}{R_{m}} .
$$

Moreover, (3) reduces to the following form:

$$
\frac{d \widetilde{P}}{d \widetilde{t}}=\beta \widetilde{P}(1-\widetilde{P})-\frac{\widetilde{Z} \widetilde{P}^{2}}{v^{2}+\widetilde{P}^{2}}, \quad \frac{d \widetilde{Z}}{d \widetilde{t}}=\gamma \widetilde{Z}\left(\frac{\widetilde{P}^{2}}{v^{2}+\widetilde{P}^{2}}-\omega\right)
$$

by using transformation

$$
\nu=\frac{\alpha}{K}, \quad \beta=\frac{r}{R_{m}}, \quad \omega=\frac{\mu}{\gamma R_{m}} .
$$

Finally, on dropping tildes, the continuous-time phytoplankton-zooplankton model (5) becomes of the following form:

$$
\frac{d P}{d t}=\beta P(1-P)-\frac{Z P^{2}}{v^{2}+P^{2}}, \quad \frac{d Z}{d t}=\gamma Z\left(\frac{P^{2}}{v^{2}+P^{2}}-\omega\right)
$$

It is well known that discrete-time models described by difference equations are more sensible than the continuous time models when populations have non-overlapping eras. Besides, discrete-time models also give more proficient computational results for numerical simulations and give a rich dynamics as compared to the continuous ones. Our extensive numerical simulations clearly show that the discrete system shows much more complex behavior as compared to the continuous one. Moreover, in spite of the fact that there are so many living circumstances in which it is characteristic to discover an occasion in discrete-time intervals, e.g., the propagation plan of phytoplankton-zooplankton. Subsequently, in order to show more reasonable and related to the living circumstance, 
we consider here a discrete form. So, by the forward Euler scheme, the discrete analogue of phytoplankton-zooplankton model (7) takes the following form:

$$
P_{n+1}=(1+h \beta) P_{n}-h\left(\beta+\frac{Z_{n}}{v^{2}+P_{n}^{2}}\right) P_{n}^{2}, \quad Z_{n+1}=Z_{n}+h \gamma Z_{n}\left(\frac{P_{n}^{2}}{v^{2}+P_{n}^{2}}-\omega\right),
$$

where $h$ denotes the step-size. Our main contributions in this article include:

- Topological classifications around fixed points of phytoplankton-zooplankton model (8).

- Exploration of periodic points of phytoplankton-zooplankton model (8).

- Comprehensive bifurcation analysis around fixed points by bifurcation theory.

- Investigation of chaos by the feedback control method for phytoplankton-zooplankton model (8).

- Validation of the obtained results numerically.

The next section is about the study of fixed points along with a linearized form of phytoplankton-zooplankton model (8), whereas topological classifications around fixed points are briefly studied in Sect. 3. Section 4 is purely dedicated to the explanation of periodic points of phytoplankton-zooplankton model (8). The comprehensive bifurcation analysis around fixed points is given in Sect. 5. Section 6 is about the investigation of chaos by the feedback control method for phytoplankton-zooplankton model (8). Theoretical results are numerically verified in Sect. 7 , whereas conclusion of the paper is given in Sect. 8.

\section{Fixed points along with linearized form of phytoplankton-zooplankton model (8)}

In the present section, the existence of fixed points along with a linearized form of phytoplankton-zooplankton model (8) are given. In the following lemma, we first summarize the result regarding the existence of fixed points of phytoplankton-zooplankton model (8) in the region $\mathbb{R}_{+}^{2}=\{(P, Z): P, Z \geq 0\}$ as follows.

Lemma 2.1 In the allowed region $\mathbb{R}_{+}^{2}=\{(P, Z): P, Z \geq 0\}$, phytoplankton-zooplankton model (8) has at most three fixed points. Specifically,

(i) Model (8) has trivial and semitrivial fixed points $F_{00}(0,0)$ and $F_{P 0}(1,0)$, respectively, $\forall \gamma, \beta, \omega, v, h$;

(ii) Model (8) has an interior fixed point $F_{P Z}^{+}\left(\sqrt{\frac{\omega \nu^{2}}{1-\omega}}, \frac{\beta v\left(\sqrt{1-\omega}-\sqrt{\omega \nu^{2}}\right)}{\sqrt{\omega}(1-\omega)}\right)$ if $\omega<\min \left\{1, \frac{1}{v^{2}+1}\right\}$.

Proof If phytoplankton-zooplankton model (8) has a fixed point $(P, Z)$, then

$$
P=(1+h \beta) P-h\left(\beta+\frac{Z}{v^{2}+P^{2}}\right) P^{2}, \quad Z=Z+h \gamma Z\left(\frac{P^{2}}{v^{2}+P^{2}}-\omega\right) .
$$

From (9), after some manipulation, one gets $P=\sqrt{\frac{\omega \nu^{2}}{1-\omega}}, Z=\frac{\beta \nu\left(\sqrt{1-\omega}-\sqrt{\omega \nu^{2}}\right)}{\sqrt{\omega}(1-\omega)}$. This gives the fact that if $\omega<\min \left\{1, \frac{1}{v^{2}+1}\right\}$, then $F_{P Z}^{+}\left(\sqrt{\frac{\omega \nu^{2}}{1-\omega}}, \frac{\beta \nu\left(\sqrt{1-\omega}-\sqrt{\omega \nu^{2}}\right)}{\sqrt{\omega}(1-\omega)}\right)$ is an interior fixed point of phytoplankton-zooplankton model (8). Additionally, $(P, Z)=(0,0),(1,0)$ satisfies (9) obviously for all parametric values $\gamma, \beta, \omega, v, h$. Therefore, $F_{00}(0,0)$ and $F_{P 0}(1,0)$ are respectively trivial and semitrivial fixed points of model (8). 
Now around the fixed point $F_{P Z}(P, Z)$ the linearized form of (8) is given for the completion of this section. For this, one has the map

$$
\left(f_{1}, f_{2}\right) \mapsto\left(P_{n+1}, Z_{n+1}\right)
$$

where

$$
f_{1}=(1+h \beta) P-h\left(\beta+\frac{Z}{v^{2}+P^{2}}\right) P^{2}, \quad f_{2}=Z+h \gamma Z\left(\frac{P^{2}}{v^{2}+P^{2}}-\omega\right)
$$

Hence the variation matrix $\left.V\right|_{F_{P Z}(P, Z)}$ around $F_{P Z}(P, Z)$ with respect to the map (10) is

$$
\left.V\right|_{F_{P Z}(P, Z)}=\left(\begin{array}{cc}
1+h \beta-2 P h\left(\beta+\frac{Z}{v^{2}+P^{2}}-\frac{P^{2} Z}{\left(v^{2}+P^{2}\right)^{2}}\right) & -\frac{h P^{2}}{v^{2}+P^{2}} \\
\frac{2 \gamma Z v^{2}}{\left(v^{2}+P^{2}\right)^{2}} & 1+h \gamma\left(\frac{P^{2}}{v^{2}+P^{2}}-\omega\right)
\end{array}\right) .
$$

\section{Topological classifications around fixed points}

The topological classifications around fixed points $F_{00}(0,0), F_{P 0}(1,0)$, and $F_{P Z}^{+}\left(\sqrt{\frac{\omega \nu^{2}}{1-\omega}}\right.$, $\left.\frac{\beta v\left(\sqrt{1-\omega}-\sqrt{\omega \nu^{2}}\right)}{\sqrt{\omega}(1-\omega)}\right)$ of model (8) are studied in this section.

\subsection{Topological classifications around $F_{00}(0,0)$}

Around $F_{00}(0,0)$, (12) becomes

$$
\left.V\right|_{F_{00}(0,0)}=\left(\begin{array}{cc}
1+h \beta & 0 \\
0 & 1-h \gamma \omega
\end{array}\right)
$$

with

$$
\lambda_{1}=1+h \beta, \quad \lambda_{2}=1-h \gamma \omega .
$$

Hence, based on stability theory, the topological classifications around $F_{00}(0,0)$ are summarized as follows.

Lemma 3.1 For $F_{00}(0,0)$, the following topological classifications hold:

(i) For allowed parametric values $\gamma, \beta, \omega, v$, and $h, F_{00}(0,0)$ is never a sink;

(ii) $F_{00}(0,0)$ is a source if

$$
\gamma>\frac{2}{h \omega}
$$

(iii) $F_{00}(0,0)$ is a saddle if

$$
0<\gamma<\frac{2}{h \omega}
$$

(iv) $F_{00}(0,0)$ is non-hyperbolic if

$$
\gamma:=\frac{2}{h \omega} .
$$




\subsection{Topological classifications around $F_{P 0}(1,0)$}

Around $F_{P 0}(1,0)$, (12) becomes

$$
\left.V\right|_{F_{P 0}(1,0)}=\left(\begin{array}{cc}
1-h \beta & \frac{-h}{v^{2}+1} \\
0 & 1+h \gamma\left(\frac{1}{v^{2}+1}-\omega\right)
\end{array}\right)
$$

with

$$
\lambda_{1}=1-h \beta, \quad \lambda_{2}=1+h \gamma\left(\frac{1}{v^{2}+1}-\omega\right) .
$$

Moreover, the topological classifications around $F_{P 0}(1,0)$ are summarized as follows.

Lemma 3.2 For $F_{P 0}(1,0)$, the following topological classifications hold:

(i) $F_{P 0}(1,0)$ is a sink if

$$
\frac{2\left(v^{2}+1\right)}{\gamma\left(\omega\left(v^{2}+1\right)-1\right)}<h<\frac{2}{\beta}
$$

(ii) $F_{P 0}(1,0)$ is a source if

$$
\frac{2\left(v^{2}+1\right)}{\gamma\left(\omega\left(v^{2}+1\right)-1\right)}>h>\frac{2}{\beta}
$$

(iii) $F_{P 0}(1,0)$ is a saddle if

$$
h>\max \left\{\frac{2}{\beta}, \frac{2\left(v^{2}+1\right)}{\gamma\left(\omega\left(v^{2}+1\right)-1\right)}\right\}
$$

(iv) $F_{P 0}(1,0)$ is non-hyperbolic if

$$
h:=\frac{2}{\beta}
$$

or

$$
h:=\frac{2\left(v^{2}+1\right)}{\gamma\left(\omega\left(v^{2}+1\right)-1\right)} .
$$

\subsection{Topological classifications around $F_{P Z}^{+}\left(\sqrt{\frac{\omega v^{2}}{1-\omega}}, \frac{\beta v\left(\sqrt{1-\omega}-\sqrt{\omega v^{2}}\right)}{\sqrt{\omega}(1-\omega)}\right)$}

Around $F_{P Z}^{+}\left(\sqrt{\frac{\omega \nu^{2}}{1-\omega}}, \frac{\beta \nu\left(\sqrt{1-\omega}-\sqrt{\omega \nu^{2}}\right)}{\sqrt{\omega}(1-\omega)}\right),(12)$ becomes

$$
\left.V\right|_{F_{P Z}^{+}\left(\sqrt{\frac{\omega \nu^{2}}{1-\omega}}, \frac{\beta \nu\left(\sqrt{1-\omega}-\sqrt{\omega \nu^{2}}\right)}{\sqrt{\omega}(1-\omega)}\right)}=\left(\begin{array}{cc}
\frac{\sqrt{1-\omega}(1-h \beta)+2 h \beta \omega\left(\sqrt{1-\omega}-\sqrt{\omega \nu^{2}}\right)}{\sqrt{1-\omega}} & -h \omega \\
2 h \beta \gamma \sqrt{1-\omega}\left(\sqrt{1-\omega}-\sqrt{\omega \nu^{2}}\right) & 1
\end{array}\right) .
$$

Further characteristic equation of (25) is

$$
\begin{aligned}
& \lambda^{2}-p\left(\sqrt{\frac{\omega \nu^{2}}{1-\omega}}, \frac{\beta \nu\left(\sqrt{1-\omega}-\sqrt{\omega \nu^{2}}\right)}{\sqrt{\omega}(1-\omega)}\right) \lambda \\
& +q\left(\sqrt{\frac{\omega \nu^{2}}{1-\omega}}, \frac{\beta \nu\left(\sqrt{1-\omega}-\sqrt{\omega \nu^{2}}\right)}{\sqrt{\omega}(1-\omega)}\right)=0,
\end{aligned}
$$


where

$$
\begin{aligned}
& p\left(\sqrt{\frac{\omega \nu^{2}}{1-\omega}}, \frac{\beta \nu\left(\sqrt{1-\omega}-\sqrt{\omega \nu^{2}}\right)}{\sqrt{\omega}(1-\omega)}\right)=\frac{\sqrt{1-\omega}(2-h \beta)+2 h \beta \omega\left(\sqrt{1-\omega}-\sqrt{\omega \nu^{2}}\right)}{\sqrt{1-\omega}}, \\
& q\left(\sqrt{\frac{\omega \nu^{2}}{1-\omega}}, \frac{\beta \nu\left(\sqrt{1-\omega}-\sqrt{\omega \nu^{2}}\right)}{\sqrt{\omega}(1-\omega)}\right) \\
& =\frac{\sqrt{1-\omega}(1-h \beta)+2 h \beta \omega\left(\sqrt{1-\omega}-\sqrt{\omega \nu^{2}}\right)(1+\gamma h(1-\omega))}{\sqrt{1-\omega}} .
\end{aligned}
$$

Finally, the roots of (26) are

$$
\lambda_{1,2}=\frac{p\left(\sqrt{\frac{\omega \nu^{2}}{1-\omega}}, \frac{\beta \nu\left(\sqrt{1-\omega}-\sqrt{\omega \nu^{2}}\right)}{\sqrt{\omega}(1-\omega)}\right) \pm \sqrt{\Delta}}{2},
$$

where

$$
\begin{aligned}
\Delta= & \left(p\left(\sqrt{\frac{\omega \nu^{2}}{1-\omega}}, \frac{\beta \nu\left(\sqrt{1-\omega}-\sqrt{\omega \nu^{2}}\right)}{\sqrt{\omega}(1-\omega)}\right)\right)^{2}-4 q\left(\sqrt{\frac{\omega \nu^{2}}{1-\omega}}, \frac{\beta \nu\left(\sqrt{1-\omega}-\sqrt{\omega \nu^{2}}\right)}{\sqrt{\omega}(1-\omega)}\right) \\
= & \left(\frac{\sqrt{1-\omega}(2-h \beta)+2 h \beta \omega\left(\sqrt{1-\omega}-\sqrt{\omega \nu^{2}}\right)}{\sqrt{1-\omega}}\right)^{2} \\
& -4\left(\frac{\sqrt{1-\omega}(1-h \beta)+2 h \beta \omega\left(\sqrt{1-\omega}-\sqrt{\omega \nu^{2}}\right)(1+\gamma h(1-\omega))}{\sqrt{1-\omega}}\right) .
\end{aligned}
$$

Hereafter the following two lemmas give the complete topological classifications around an interior fixed point $F_{P Z}^{+}\left(\sqrt{\frac{\omega \nu^{2}}{1-\omega}}, \frac{\beta \nu\left(\sqrt{1-\omega}-\sqrt{\omega \nu^{2}}\right)}{\sqrt{\omega}(1-\omega)}\right)$ of phytoplankton-zooplankton model (8) if $\Delta<0$ and $\Delta \geq 0$, respectively.

Lemma 3.3 If $\Delta=\left(\frac{\sqrt{1-\omega}(2-h \beta)+2 h \beta \omega\left(\sqrt{1-\omega}-\sqrt{\omega \nu^{2}}\right)}{\sqrt{1-\omega}}\right)^{2}-4\left(\frac{\sqrt{1-\omega}(1-h \beta)+2 h \beta \omega\left(\sqrt{1-\omega}-\sqrt{\omega \nu^{2}}\right)(1+\gamma h(1-\omega))}{\sqrt{1-\omega}}\right)<$ 0 , then around $F_{P Z}^{+}\left(\sqrt{\frac{\omega \nu^{2}}{1-\omega}}, \frac{\beta v\left(\sqrt{1-\omega}-\sqrt{\omega \nu^{2}}\right)}{\sqrt{\omega}(1-\omega)}\right)$ of phytoplankton-zooplankton model (8), the following topological classifications hold:

(i) $F_{P Z}^{+}\left(\sqrt{\frac{\omega \nu^{2}}{1-\omega}}, \frac{\beta v\left(\sqrt{1-\omega}-\sqrt{\omega \nu^{2}}\right)}{\sqrt{\omega}(1-\omega)}\right)$ is a stable focus if

$$
0<\gamma<\frac{\sqrt{1-\omega}-2 \omega\left(\sqrt{1-\omega}-\sqrt{\omega \nu^{2}}\right)}{2 h \omega(1-\omega)\left(\sqrt{1-\omega}-\sqrt{\omega \nu^{2}}\right)}
$$

with

$$
v<\frac{1-2 \omega}{2 \omega} \sqrt{\frac{1-\omega}{\omega}}
$$

(ii) $F_{P Z}^{+}\left(\sqrt{\frac{\omega \nu^{2}}{1-\omega}}, \frac{\beta v\left(\sqrt{1-\omega}-\sqrt{\omega \nu^{2}}\right)}{\sqrt{\omega}(1-\omega)}\right)$ is an unstable focus if (31) holds and

$$
\gamma>\frac{\sqrt{1-\omega}-2 \omega\left(\sqrt{1-\omega}-\sqrt{\omega \nu^{2}}\right)}{2 h \omega(1-\omega)\left(\sqrt{1-\omega}-\sqrt{\omega \nu^{2}}\right)}
$$


(iii) $P_{P Z}^{+}\left(\sqrt{\frac{\omega \nu^{2}}{1-\omega}}, \frac{\beta \nu\left(\sqrt{1-\omega}-\sqrt{\omega \nu^{2}}\right)}{\sqrt{\omega}(1-\omega)}\right)$ is non-hyperbolic if

$$
\gamma:=\frac{\sqrt{1-\omega}-2 \omega\left(\sqrt{1-\omega}-\sqrt{\omega \nu^{2}}\right)}{2 h \omega(1-\omega)\left(\sqrt{1-\omega}-\sqrt{\omega \nu^{2}}\right)} .
$$

Lemma 3.4 If $\Delta=\left(\frac{\sqrt{1-\omega}(2-h \beta)+2 h \beta \omega\left(\sqrt{1-\omega}-\sqrt{\omega \nu^{2}}\right)}{\sqrt{1-\omega}}\right)^{2}-4\left(\frac{\sqrt{1-\omega}(1-h \beta)+2 h \beta \omega\left(\sqrt{1-\omega}-\sqrt{\omega \nu^{2}}\right)(1+\gamma h(1-\omega))}{\sqrt{1-\omega}}\right) \geq$ 0 , then around $F_{P Z}^{+}\left(\sqrt{\frac{\omega v^{2}}{1-\omega}}, \frac{\beta v\left(\sqrt{1-\omega}-\sqrt{\omega v^{2}}\right)}{\sqrt{\omega}(1-\omega)}\right)$ of phytoplankton-zooplankton model (8), the following topological classifications hold:

(i) $F_{p z}^{+}\left(\sqrt{\frac{\omega \nu^{2}}{1-\omega}}, \frac{\beta \nu\left(\sqrt{1-\omega}-\sqrt{\omega \nu^{2}}\right)}{\sqrt{\omega}(1-\omega)}\right)$ is a stable node if

$$
0<\gamma<\frac{(h \beta-2) \sqrt{1-\omega}-2 h \beta \omega\left(\sqrt{1-\omega}-\sqrt{\omega \nu^{2}}\right)}{h^{2} \beta \omega(1-\omega)\left(\sqrt{1-\omega}-\sqrt{\omega \nu^{2}}\right)}
$$

with

$$
h>\frac{2 \sqrt{1-\omega}}{\beta\left((\sqrt{1-\omega})(1-\omega)+\omega \sqrt{\omega \nu^{2}}\right)}
$$

(ii) $F_{P Z}^{+}\left(\sqrt{\frac{\omega v^{2}}{1-\omega}}, \frac{\beta \nu\left(\sqrt{1-\omega}-\sqrt{\omega \nu^{2}}\right)}{\sqrt{\omega}(1-\omega)}\right)$ is an unstable node if (35) holds and

$$
\gamma>\frac{(h \beta-2) \sqrt{1-\omega}-2 h \beta \omega\left(\sqrt{1-\omega}-\sqrt{\omega \nu^{2}}\right)}{h^{2} \beta \omega(1-\omega)\left(\sqrt{1-\omega}-\sqrt{\omega \nu^{2}}\right)}
$$

(iii) $F_{P Z}^{+}\left(\sqrt{\frac{\omega \nu^{2}}{1-\omega}}, \frac{\beta v\left(\sqrt{1-\omega}-\sqrt{\omega \nu^{2}}\right)}{\sqrt{\omega}(1-\omega)}\right)$ is non-hyperbolic if

$$
\gamma:=\frac{(h \beta-2) \sqrt{1-\omega}-2 h \beta \omega\left(\sqrt{1-\omega}-\sqrt{\omega \nu^{2}}\right)}{h^{2} \beta \omega(1-\omega)\left(\sqrt{1-\omega}-\sqrt{\omega \nu^{2}}\right)} .
$$

\section{Periodic points of phytoplankton-zooplankton model (8)}

Now it is proved that fixed points $F_{00}(0,0), F_{P 0}(1,0)$, and $F_{P Z}^{+}\left(\sqrt{\frac{\omega \nu^{2}}{1-\omega}}, \frac{\beta \nu\left(\sqrt{1-\omega}-\sqrt{\omega \nu^{2}}\right)}{\sqrt{\omega}(1-\omega)}\right)$ of phytoplankton-zooplankton model (8) are periodic points with period- $2,3, \ldots, n$.

Theorem 4.1 $F_{00}(0,0)$ of phytoplankton-zooplankton model (8) is a periodic point of prime period-1.

Proof From (8) denote

$$
\Phi:=\left(f_{1}, f_{2}\right)
$$

where $f_{1}$ and $f_{2}$ are depicted in (11). After straightforward computation, from (38) one gets the following desired statement:

$$
\left.\Phi\right|_{F_{00}(0,0)}=F_{00}(0,0)
$$

In a similar way, one can prove that the rest of fixed points $F_{P 0}(1,0)$ and $F_{P Z}^{+}\left(\sqrt{\frac{\omega v^{2}}{1-\omega}}\right.$, $\left.\frac{\beta \nu\left(\sqrt{1-\omega}-\sqrt{\omega \nu^{2}}\right)}{\sqrt{\omega}(1-\omega)}\right)$ of phytoplankton-zooplankton model (8) are periodic points of prime period-1. 


\section{Theorem 4.2}

(i) $F_{P 0}(1,0)$ of phytoplankton-zooplankton model (8) is a periodic point of prime period-1;

(ii) $F_{P Z}^{+}\left(\sqrt{\frac{\omega \nu^{2}}{1-\omega}}, \frac{\beta v\left(\sqrt{1-\omega}-\sqrt{\omega \nu^{2}}\right)}{\sqrt{\omega}(1-\omega)}\right)$ of phytoplankton-zooplankton model (8) is a periodic point of prime period-1.

Proof Same as the proof of Theorem 4.1 .

Now we will investigate that the fixed points $F_{00}(0,0), F_{P 0}(1,0)$, and $F_{P Z}^{+}\left(\sqrt{\frac{\omega v^{2}}{1-\omega}}\right.$, $\left.\frac{\beta \nu\left(\sqrt{1-\omega}-\sqrt{\omega \nu^{2}}\right)}{\sqrt{\omega}(1-\omega)}\right)$ of phytoplankton-zooplankton model (8) are periodic points with period$2,3, \ldots, n$.

\section{Theorem 4.3}

(i) $F_{00}(0,0)$ of phytoplankton-zooplankton model (8) is a periodic point of period $-2,3, \ldots, n$;

(ii) $F_{P 0}(1,0)$ of phytoplankton-zooplankton model (8) is a periodic point of period $-2,3, \ldots, n$

(iii) $F_{P Z}^{+}\left(\sqrt{\frac{\omega v^{2}}{1-\omega}}, \frac{\beta v\left(\sqrt{1-\omega}-\sqrt{\omega v^{2}}\right)}{\sqrt{\omega}(1-\omega)}\right)$ of phytoplankton-zooplankton model (8) is a periodic point of period $-2,3, \ldots, n$.

Proof (i) In view of (38) we have

$$
\left.\begin{aligned}
\Phi^{2}= & \left((1+h \beta) f_{1}-h\left(\beta+\frac{f_{2}}{v^{2}+f_{1}^{2}}\right) f_{1}^{2},\right. \\
& \left.f_{2}+h \gamma f_{2}\left(\frac{f_{1}^{2}}{v^{2}+\left(f_{1}\right)^{2}}-\omega\right)\right) \\
\Phi^{3}= & \left((1+h \beta) f_{1}^{2}-h\left(\beta+\frac{f_{2}^{2}}{v^{2}+\left(f_{1}^{2}\right)^{2}}\right)\left(f_{1}^{2}\right)^{2},\right.
\end{aligned} \quad \Rightarrow \Phi^{2}\right|_{F_{00}(0,0)}=F_{00}(0,0),
$$

Therefore from (40) one has the required result.

(ii) In view of proof (i) of Theorem 4.3, one gets the required statement:

$$
\begin{gathered}
\Phi_{F_{P 0}(1,0)}^{2}=F_{P 0}(1,0), \\
\Phi_{F_{P 0}(1,0)}^{3}=F_{P 0}(1,0), \\
\vdots \\
\Phi_{F_{P 0}(1,0)}^{i}=F_{P 0}(1,0) .
\end{gathered}
$$


(iii) The following computation shows the required statement:

$$
\begin{gathered}
\Phi_{F_{P Z}^{+}\left(\sqrt{\frac{\omega \nu^{2}}{1-\omega}}, \frac{\beta \nu\left(\sqrt{1-\omega}-\sqrt{\omega \nu^{2}}\right)}{\sqrt{\omega}(1-\omega)}\right)}^{2}=F_{P Z}^{+}\left(\sqrt{\frac{\omega \nu^{2}}{1-\omega}}, \frac{\beta \nu\left(\sqrt{1-\omega}-\sqrt{\omega \nu^{2}}\right)}{\sqrt{\omega}(1-\omega)}\right), \\
\Phi_{F_{P Z}^{+}\left(\sqrt{\frac{\omega \nu^{2}}{1-\omega}}, \frac{\beta \nu\left(\sqrt{1-\omega}-\sqrt{\omega \nu^{2}}\right)}{\sqrt{\omega}(1-\omega)}\right)}^{3}=F_{P Z}^{+}\left(\sqrt{\frac{\omega \nu^{2}}{1-\omega}}, \frac{\beta \nu\left(\sqrt{1-\omega}-\sqrt{\omega \nu^{2}}\right)}{\sqrt{\omega}(1-\omega)}\right), \\
\vdots \\
\Phi_{F_{P Z}^{+}\left(\sqrt{\frac{\omega \nu^{2}}{1-\omega}}, \frac{\beta \nu\left(\sqrt{1-\omega}-\sqrt{\omega \nu^{2}}\right)}{\sqrt{\omega(1-\omega)}}\right)}^{i}=F_{P Z}^{+}\left(\sqrt{\frac{\omega \nu^{2}}{1-\omega}}, \frac{\beta \nu\left(\sqrt{1-\omega}-\sqrt{\omega \nu^{2}}\right)}{\sqrt{\omega}(1-\omega)}\right) .
\end{gathered}
$$

\section{Bifurcation analysis}

The full and comprehensive possible bifurcation analysis of phytoplankton-zooplankton model (8) around fixed points $F_{00}(0,0), F_{P 0}(1,0), F_{P Z}^{+}\left(\sqrt{\frac{\omega \nu^{2}}{1-\omega}}, \frac{\beta v\left(\sqrt{1-\omega}-\sqrt{\omega \nu^{2}}\right)}{\sqrt{\omega}(1-\omega)}\right)$ will be investigated based on theoretical studies in Sect. 3 by bifurcation theory [8-21].

\subsection{Bifurcation analysis around $F_{00}(0,0)$}

In the following, we prove that around $F_{00}(0,0)$, the occurrence of possible bifurcations is flip bifurcation and transcritical bifurcation.

\subsubsection{Flip analysis around $F_{00}(0,0)$}

If (17) holds, then from (14) one gets $\left.\lambda_{2}\right|_{(17)}=-1$, but $\left.\lambda_{1}\right|_{(17)}=1+\frac{2 \beta}{\gamma \omega} \neq 1$ or -1 . This infers the fact that phytoplankton-zooplankton model (8) may undergo a flip bifurcation by choosing $\gamma$ as a bifurcation parameter if $(\gamma, h, \beta, \omega, \nu)$ is located in the set

$$
\left.\mathcal{F}\right|_{F_{00}(0,0)}:=\left\{(\gamma, h, \beta, \omega, \nu), \gamma=\frac{2}{h \omega}\right\}
$$

where $\left.\mathcal{F}\right|_{F_{00}(0,0)}$ stands for the flip bifurcation around the fixed point $F_{00}(0,0)$. But computation shows that this bifurcation cannot occur if $\left.(\gamma, h, \beta, \omega, v) \in \mathcal{F}\right|_{F_{00}(0,0)}$, and therefore the fixed point $F_{00}(0,0)$ is degenerate with higher co-dimension.

\subsubsection{Transcritical bifurcation around $F_{00}(0,0)$}

In the following theorem we prove that around $F_{00}(0,0)$, phytoplankton-zooplankton model (8) undergoes a transcritical bifurcation by choosing $\gamma$ as a bifurcation parameter.

Theorem 5.1 Around $F_{00}(0,0)$, phytoplankton-zooplankton model (8) undergoes a transcritical bifurcation if $(\gamma, h, \beta, \omega, v)$ goes through the following curve:

$$
\left.\mathcal{T}\right|_{F_{00}(0,0)}:=\left\{(\gamma, h, \beta, \omega, \nu), \gamma=\frac{2}{\omega h}\right\} .
$$

Proof Under the same manipulation as we have done in Sect. 5.1.1, it is recalled that $F_{00}(0,0)$ is non-hyperbolic if $(17)$ holds. So, by choosing $\gamma$ as a bifurcation parameter, the 
central manifold of the following map

$$
P_{n+1}=(1+h \beta) P_{n}-h \beta P_{n}^{2}
$$

is $Z=0$, where (17) holds. Thus it manifests that phytoplankton-zooplankton model (8) undergoes a transcritical bifurcation at $F_{00}(0,0)$.

\subsection{Bifurcation analysis around $F_{P 0}(1,0)$}

If (23) holds, then from (19) one gets $\left.\lambda_{1}\right|_{(23)}=-1$, but $\left.\lambda_{2}\right|_{(23)}=1+\frac{2 \gamma}{\beta}\left(\frac{1}{v^{2}+1}-\omega\right) \neq 1$ or -1 . This gives the fact that phytoplankton-zooplankton model (8) may undergo a flip bifurcation by choosing $h$ as a bifurcation parameter if $(\gamma, h, \beta, \omega, v)$ is located in the set

$$
\left.\mathcal{F}\right|_{F_{P 0}(1,0)}:=\left\{(\gamma, h, \beta, \omega, \nu), h=\frac{2}{\beta}\right\} .
$$

But the following result shows the fact that the flip bifurcation does not exist around $F_{P 0}(1,0)$ of phytoplankton-zooplankton model $(8)$.

Theorem 5.2 If $\left.(\gamma, h, \beta, \omega, v) \in \mathcal{F}\right|_{F_{P 0}(1,0)}$, then there exists no flip bifurcation around $F_{P 0}(1,0)$ of phytoplankton-zooplankton model $(8)$.

Proof It is noted here that phytoplankton-zooplankton model (8) is invariant with respect to $Z=0$, and in order to explore the said bifurcation, we restrict the model on the line $Z=0$, where (8) takes the form

$$
P_{n+1}=(1+h \beta) P_{n}-h \beta P_{n}^{2} .
$$

From (47) one has the following one-dimensional map with $h$ as a bifurcation parameter:

$$
f(h, P):=(1+h \beta) P-h \beta P^{2} .
$$

Finally, if $h=h^{*}=\frac{2}{\beta}$ and $P=P^{*}=1$, then from (48) one gets:

$$
\begin{aligned}
& \left.\frac{\partial f}{\partial P}\right|_{h^{*}=\frac{2}{\beta}, P^{*}=1}:=-1, \\
& \left.\frac{\partial^{2} f}{\partial P^{2}}\right|_{h^{*}=\frac{2}{\beta}, P^{*}=1}:=-4 \neq 0,
\end{aligned}
$$

and

$$
\left.\frac{\partial f}{\partial h}\right|_{h^{*}=\frac{2}{\beta}, P^{*}=1}:=0 .
$$

The computed condition, which is depicted in (51), violates the nondegenerate condition, and hence this implies the fact that if $\left.(\gamma, h, \beta, \omega, v) \in \mathcal{F}\right|_{F_{P 0}(1,0)}$, then there exists no flip bifurcation around $F_{P 0}(1,0)$ of phytoplankton-zooplankton model (8). 
5.3 Bifurcation analysis around $F_{P Z}^{+}\left(\sqrt{\frac{\omega \nu^{2}}{1-\omega}}, \frac{\beta \nu\left(\sqrt{1-\omega}-\sqrt{\omega \nu^{2}}\right)}{\sqrt{\omega}(1-\omega)}\right)$

In the following, we prove that around $F_{P Z}^{+}\left(\sqrt{\frac{\omega \nu^{2}}{1-\omega}}, \frac{\beta v\left(\sqrt{1-\omega}-\sqrt{\omega \nu^{2}}\right)}{\sqrt{\omega}(1-\omega)}\right)$ the occurrence of possible bifurcations are Neimark-Sacker and flip bifurcations.

5.3.1 Neimark-Sacker bifurcation around $F_{P Z}^{+}\left(\sqrt{\frac{\omega \nu^{2}}{1-\omega}}, \frac{\beta \nu\left(\sqrt{1-\omega}-\sqrt{\omega \nu^{2}}\right)}{\sqrt{\omega(1-\omega)}}\right)$

If (33) holds, then the variational matrix $\left.V\right|_{F_{P Z}^{+}\left(\sqrt{\frac{\omega \nu^{2}}{1-\omega}}, \frac{\beta v\left(\sqrt{1-\omega}-\sqrt{\omega \nu^{2}}\right)}{\sqrt{\omega}(1-\omega)}\right)}$ around $F_{P Z}^{+}\left(\sqrt{\frac{\omega \nu^{2}}{1-\omega}}\right.$, $\left.\frac{\beta \nu\left(\sqrt{1-\omega}-\sqrt{\omega \nu^{2}}\right)}{\sqrt{\omega}(1-\omega)}\right)$ has complex eigenvalues with $\left|\lambda_{1,2}\right|_{(33)}=1$, which further shows that around $F_{P Z}^{+}\left(\sqrt{\frac{\omega \nu^{2}}{1-\omega}}, \frac{\beta \nu\left(\sqrt{1-\omega}-\sqrt{\omega \nu^{2}}\right)}{\sqrt{\omega}(1-\omega)}\right)$ there may exist the N-S bifurcation by choosing $\gamma$ as a bifurcation parameter if $(\gamma, h, \beta, \omega, v)$ are located in the set

$$
\left.\mathcal{N}\right|_{F_{P Z}^{+}\left(\sqrt{\frac{\omega v^{2}}{1-\omega}}, \frac{\beta \nu\left(\sqrt{1-\omega}-\sqrt{\omega \nu^{2}}\right)}{\sqrt{\omega}(1-\omega)}\right)}:=\left\{(\gamma, h, \beta, \omega, \nu), \gamma=\frac{\sqrt{1-\omega}-2 \omega\left(\sqrt{1-\omega}-\sqrt{\omega \nu^{2}}\right)}{2 h \omega(1-\omega)\left(\sqrt{1-\omega}-\sqrt{\omega \nu^{2}}\right)}\right\} .
$$

But the following theorem guarantees the fact that around $F_{P Z}^{+}\left(\sqrt{\frac{\omega v^{2}}{1-\omega}}, \frac{\beta v\left(\sqrt{1-\omega}-\sqrt{\omega \nu^{2}}\right)}{\sqrt{\omega}(1-\omega)}\right)$ phytoplankton-zooplankton model (8) must undergo the Neimark-Sacker bifurcation.

Theorem 5.3 If $\left.(\gamma, h, \beta, \omega, v) \in \mathcal{N}\right|_{F_{P Z}^{+}\left(\sqrt{\frac{\omega \nu^{2}}{1-\omega}}, \frac{\beta v\left(\sqrt{1-\omega}-\sqrt{\omega \nu^{2}}\right)}{\sqrt{\omega}(1-\omega)}\right)}$ then around $F_{P Z}^{+}\left(\sqrt{\frac{\omega \nu^{2}}{1-\omega}}\right.$, $\left.\frac{\beta \nu\left(\sqrt{1-\omega}-\sqrt{\omega \nu^{2}}\right)}{\sqrt{\omega}(1-\omega)}\right)$ phytoplankton-zooplankton model (8) undergoes the Neimark-Sacker bifurcation.

Proof Since $\left.(\gamma, h, \beta, \omega, v) \in \mathcal{N}\right|_{F_{P Z}^{+}\left(\sqrt{\frac{\omega \nu^{2}}{1-\omega}}, \frac{\beta \nu\left(\sqrt{1-\omega}-\sqrt{\omega \nu^{2}}\right)}{\sqrt{\omega}}\right)}$, it is clear that $\gamma$ is the bifurcation parameter. Therefore if $\gamma$ is a nbhd of $\gamma^{*}$, i.e., $\gamma=\gamma^{*}+\epsilon$, where $\epsilon \ll 1$, then phytoplanktonzooplankton model (8) takes the following form:

$$
P_{n+1}=(1+h \beta) P_{n}-h\left(\beta+\frac{Z_{n}}{v^{2}+P_{n}^{2}}\right) P_{n}^{2}, Z_{n+1}=Z_{n}+h\left(\gamma^{*}+\epsilon\right) Z_{n}\left(\frac{P_{n}^{2}}{v^{2}+P_{n}^{2}}-\omega\right),
$$

with $F_{P Z}^{+}\left(\sqrt{\frac{\omega \nu^{2}}{1-\omega}}, \frac{\beta \nu\left(\sqrt{1-\omega}-\sqrt{\omega \nu^{2}}\right)}{\sqrt{\omega}(1-\omega)}\right)$ as an interior fixed point. Further, a pair of complex roots of the characteristic equation of variational matrix $\left.V\right|_{F_{P Z}^{+}\left(\sqrt{\frac{\omega \nu^{2}}{1-\omega}}, \frac{\beta v\left(\sqrt{1-\omega}-\sqrt{\omega \nu^{2}}\right)}{\sqrt{\omega(1-\omega)}}\right)}$ around $F_{P Z}^{+}\left(\sqrt{\frac{\omega \nu^{2}}{1-\omega}}, \frac{\beta \nu\left(\sqrt{1-\omega}-\sqrt{\omega \nu^{2}}\right)}{\sqrt{\omega}(1-\omega)}\right)$ of model (53) is

$$
\lambda_{1,2}=\frac{p(\epsilon) \pm \iota \sqrt{4 q(\epsilon)-p^{2}(\epsilon)}}{2},
$$

where

$$
\begin{aligned}
& p(\epsilon)=\frac{\sqrt{1-\omega}(2-h \beta)+2 h \omega \beta\left(\sqrt{1-\omega}-\sqrt{\omega \nu^{2}}\right)}{\sqrt{1-\omega}}, \\
& q(\epsilon)=\frac{\sqrt{1-\omega}(1-h \beta)+2 h \omega \beta\left(\sqrt{1-\omega}-\sqrt{\omega \nu^{2}}\right)\left(1+\left(\gamma^{*}+\epsilon\right) h(1-\omega)\right)}{\sqrt{1-\omega}} .
\end{aligned}
$$

In view of (54) along with (55), one gets

$$
\left|\lambda_{1,2}\right|=\sqrt{\frac{\sqrt{1-\omega}(1-\beta)+2 \omega \beta\left(\sqrt{1-\omega}-\sqrt{\omega \nu^{2}}\right)\left(1+\left(\gamma^{*}+\epsilon\right) h(1-\omega)\right)}{\sqrt{1-\omega}}}
$$


and

$$
\left.\frac{d\left|\lambda_{1,2}\right|}{d \epsilon}\right|_{\epsilon=0}=\frac{\beta\left((3-2 \omega) \sqrt{1-\omega}+2 \omega \sqrt{\omega \nu^{2}}\right)}{2 \sqrt{1-\omega}} \neq 0 .
$$

Further, for the existence of N-S bifurcation around $F_{P Z}^{+}\left(\sqrt{\frac{\omega \nu^{2}}{1-\omega}}, \frac{\beta \nu\left(\sqrt{1-\omega}-\sqrt{\omega \nu^{2}}\right)}{\sqrt{\omega}(1-\omega)}\right)$ of model (53), it is also required that $\lambda_{1,2}^{m} \neq 1, m=1,2,3,4$, if $\epsilon=0$, which is equivalent to $p(0) \neq$ $-2,0,1,2$. But if (33) holds, then from (55) one gets $q(0)=1$. Therefore $p(0) \neq-2,2$, and so it is only required that $p(0) \neq 0,1$. By computation one gets

$$
h \neq \frac{2 \sqrt{1-\omega}}{\beta\left((1-\omega)(1-2 \omega)+2 \omega \sqrt{\omega \nu^{2}}\right)}, \frac{\sqrt{1-\omega}}{\beta\left((1-\omega)(1-2 \omega)+2 \omega \sqrt{\omega \nu^{2}}\right)}
$$

Hereafter, in order to transform $F_{P Z}^{+}\left(\sqrt{\frac{\omega \nu^{2}}{1-\omega}}, \frac{\beta \nu\left(\sqrt{1-\omega}-\sqrt{\omega v^{2}}\right)}{\sqrt{\omega}(1-\omega)}\right)$ of $(53)$ to origin, i.e., $F_{00}(0,0)$, we use the following transformation:

$$
u_{n}=P_{n}-P^{*}, \quad v_{n}=Z_{n}-Z^{*},
$$

with $P^{*}=\sqrt{\frac{\omega \nu^{2}}{1-\omega}}, Z^{*}=\frac{\beta \nu\left(\sqrt{1-\omega}-\sqrt{\omega v^{2}}\right)}{\sqrt{\omega}(1-\omega)}$. In view of (59), from (53) one gets

$$
\begin{aligned}
& u_{n+1}=(1+h \beta)\left(u_{n}+P^{*}\right)-h\left(\beta+\frac{v_{n}+Z^{*}}{v^{2}+\left(u_{n}+P^{*}\right)^{2}}\right)\left(u_{n}+P^{*}\right)^{2}-P^{*}, \\
& v_{n+1}=v_{n}+Z^{*}+h\left(\gamma^{*}+\epsilon\right)\left(v_{n}+Z^{*}\right)\left(\frac{\left(u_{n}+P^{*}\right)^{2}}{v^{2}+\left(u_{n}+P^{*}\right)^{2}}-\omega\right)-Z^{*} .
\end{aligned}
$$

Now a normal form of (60) is explored if $\epsilon=0$. For this expanding (60) up to order-2nd around $F_{00}(0,0)$, one gets

$$
\begin{aligned}
& u_{n+1}=\alpha_{11} u_{n}+\alpha_{12} v_{n}+\alpha_{13} u_{n}^{2}+\alpha_{14} u_{n} v_{n}+\alpha_{15} u_{n}^{3}+\alpha_{16} u_{n}^{2} v_{n}, \\
& v_{n+1}=\alpha_{21} u_{n}+\alpha_{22} v_{n}+\alpha_{23} u_{n}^{2}+\alpha_{24} u_{n} v_{n}+\alpha_{25} u_{n}^{3}+\alpha_{26} u_{n}^{2} v_{n},
\end{aligned}
$$

with

$$
\begin{aligned}
& \alpha_{11}=1+h \beta-2 h \beta P^{*}-\frac{2 h v^{2} Z^{*} P^{*}}{\left(v^{2}+P^{* 2}\right)^{2}}, \\
& \alpha_{12}=-\frac{h P^{* 2}}{\left(v^{2}+P^{* 2}\right)^{2}}, \\
& \alpha_{13}=-h \beta-\frac{h v^{2} Z^{*}\left(v^{2}-3 P^{* 2}\right)}{\left(v^{2}+P^{* 2}\right)^{3}}, \\
& \alpha_{14}=-\frac{2 h v^{2} P^{*}}{\left(v^{2}+P^{* 2}\right)^{2}}, \\
& \alpha_{15}=\frac{4 h Z^{*} v^{2} P^{*}\left(v^{2}-P^{* 2}\right)}{\left(v^{2}+P^{* 2}\right)^{4}}, \\
& \alpha_{16}=-\frac{h v^{2}\left(v^{2}-3 P^{* 2}\right)}{\left(v^{2}+P^{* 2}\right)^{3}},
\end{aligned}
$$




$$
\begin{aligned}
& \alpha_{21}=\frac{2 h \gamma^{*} Z^{*} v^{2} P^{*}}{\left(v^{2}+P^{* 2}\right)^{2}}, \\
& \alpha_{22}=1+h \gamma^{*} Z^{*}\left(\frac{P^{* 2}}{v^{2}+P^{* 2}}-\omega\right), \\
& \alpha_{23}=h \gamma^{*} Z^{*} v^{2}\left(\frac{v^{2}-3 P^{* 2}}{\left(v^{2}+P^{* 2}\right)^{3}}\right), \\
& \alpha_{24}=\frac{2 h \gamma^{*} v^{2} P^{*}}{\left(v^{2}+P^{* 2}\right)^{2}}, \\
& \alpha_{25}=-4 h \gamma^{*} v^{2} Z^{*} P^{*}\left(\frac{v^{2}-P^{* 2}}{\left(v^{2}+P^{* 2}\right)^{4}}\right), \\
& \alpha_{26}=h \gamma^{*} v^{2}\left(\frac{v^{2}-3 P^{* 2}}{\left(v^{2}+P^{* 2}\right)^{3}}\right) .
\end{aligned}
$$

Now, we use the following transformation in order to transform the linear part of (61) into a canonical form

$$
\left(\begin{array}{l}
u_{n} \\
v_{n}
\end{array}\right)=\left(\begin{array}{cc}
\alpha_{12} & 0 \\
\eta-\alpha_{11} & -\zeta
\end{array}\right)\left(\begin{array}{c}
P_{n} \\
Z_{n}
\end{array}\right),
$$

with

$$
\begin{aligned}
& \eta= \frac{(2-h \beta) \sqrt{1-\omega}+2 h \omega \beta\left(\sqrt{1-\omega}-\sqrt{\omega \nu^{2}}\right)}{2 \sqrt{1-\omega}}, \\
& \zeta=\frac{1}{2} \sqrt{\frac{4\left((1-h \beta) \sqrt{1-\omega}+2 h \beta \omega\left(\sqrt{1-\omega}-\sqrt{\omega \nu^{2}}\right)\right)(1+h \gamma(1-\omega))}{\sqrt{1-\omega}}} \\
&-\left(\frac{\sqrt{1-\omega}(2-h \beta)+2 h \beta \omega\left(\sqrt{1-\omega}-\sqrt{\omega \nu^{2}}\right)}{\sqrt{1-\omega}}\right)^{2} .
\end{aligned}
$$

In view of (63), (61) takes the following form:

$$
\begin{aligned}
& P_{n+1}=\eta P_{n}-\zeta Z_{n}+\bar{P}\left(P_{n}, Z_{n}\right), \\
& Z_{n+1}=\zeta P_{n}+\eta Z_{n}+\bar{Q}\left(P_{n}, Z_{n}\right),
\end{aligned}
$$

where

$$
\begin{aligned}
& \bar{P}\left(P_{n}, Z_{n}\right)=r_{11} P_{n}^{3}+r_{12} P_{n}^{2}+r_{13} P_{n}^{2} Z_{n}+r_{14} P_{n} Z_{n}, \\
& \bar{Q}\left(P_{n}, Z_{n}\right)=r_{21} P_{n}^{3}+r_{22} P_{n}^{2}+r_{23} P_{n}^{2} Z_{n}+r_{24} P_{n} Z_{n},
\end{aligned}
$$

and

$$
\begin{aligned}
& r_{11}=\alpha_{15} \alpha_{12}^{2}+\alpha_{16} \alpha_{12}\left(\eta-\alpha_{11}\right), \\
& r_{12}=\alpha_{13} \alpha_{12}+\alpha_{14}\left(\eta-\alpha_{11}\right), \\
& r_{13}=-\alpha_{16} \alpha_{12} \zeta, \\
& r_{14}=-\alpha_{14} \zeta,
\end{aligned}
$$




$$
\begin{aligned}
& r_{21}=\frac{1}{\zeta}\left(\alpha_{15} \alpha_{12}\left(\eta-\alpha_{11}\right)-\alpha_{25} \alpha_{12}^{3}+\alpha_{16} \alpha_{12}\left(\eta-\alpha_{11}\right)-\alpha_{26} \alpha_{12}^{2}\left(\eta-\alpha_{11}\right)\right), \\
& r_{22}=\frac{1}{\zeta}\left(\alpha_{13} \alpha_{12}\left(\eta-\alpha_{11}\right)-\alpha_{13} \alpha_{12}^{2}+\alpha_{14}\left(\eta-\alpha_{11}\right)^{2}-\alpha_{24} \alpha_{12}\left(\eta-\alpha_{11}\right)\right), \\
& r_{23}=-\left(\alpha_{16} \alpha_{12}-\alpha_{26} \alpha_{12}^{2}\right), \\
& r_{24}=-\left(\alpha_{14}\left(\eta-\alpha_{11}\right)-\alpha_{24} \alpha_{12}\right) .
\end{aligned}
$$

From (66) one gets

$$
\begin{aligned}
& \left.\frac{\partial^{2} \bar{P}}{\partial P_{n}^{2}}\right|_{F_{00}(0,0)}=2\left(r_{12}+r_{13}\right) \text {, } \\
& \left.\frac{\partial^{2} \bar{P}}{\partial P_{n} \partial Z_{n}}\right|_{F_{00}(0,0)}=r_{14}, \\
& \left.\frac{\partial^{2} \bar{P}}{\partial Z_{n}^{2}}\right|_{F_{00}(0,0)}=0 \text {, } \\
& \left.\frac{\partial^{3} \bar{P}}{\partial P_{n}^{3}}\right|_{F_{00}(0,0)}=6 r_{11} \text {, } \\
& \left.\frac{\partial^{3} \bar{P}}{\partial P_{n}^{2} \partial Z_{n}}\right|_{F_{00}(0,0)}=0 \text {, } \\
& \left.\frac{\partial^{3} \bar{P}}{\partial P_{n} \partial Z_{n}^{2}}\right|_{F_{00}(0,0)}=0 \text {, } \\
& \left.\frac{\partial^{3} \bar{P}}{\partial Z_{n}^{3}}\right|_{F_{00}(0,0)}=0 \text {, } \\
& \left.\frac{\partial^{2} \bar{Q}}{\partial P_{n}^{2}}\right|_{F_{00}(0,0)}=2\left(r_{22}+r_{23}\right) \text {, } \\
& \left.\frac{\partial^{2} \bar{Q}}{\partial P_{n} \partial Z_{n}}\right|_{F_{00}(0,0)}=r_{24} \text {, } \\
& \left.\frac{\partial^{2} \bar{Q}}{\partial Z_{n}^{2}}\right|_{F_{00}(0,0)}=0 \text {, } \\
& \left.\frac{\partial^{3} \bar{Q}}{\partial P_{n}^{3}}\right|_{F_{00}(0,0)}=6 r_{21} \text {, } \\
& \left.\frac{\partial^{3} \bar{Q}}{\partial P_{n}^{2} \partial Z_{n}}\right|_{F_{00}(0,0)}=0 \text {. } \\
& \left.\frac{\partial^{3} \bar{Q}}{\partial P_{n} \partial Z_{n}^{2}}\right|_{F_{00}(0,0)}=0 \text {, } \\
& \left.\frac{\partial^{3} \bar{Q}}{\partial Z_{n}^{3}}\right|_{F_{00}(0,0)}=0 \text {. }
\end{aligned}
$$

Finally, to determine that (65) undergoes the N-S bifurcation, the following quantity is required to be nonzero:

$$
\Pi=-\Re\left(\frac{(1-2 \bar{\lambda}) \bar{\lambda}^{2}}{1-\lambda} \varrho_{11} \varrho_{20}\right)-\frac{1}{2}\left\|\varrho_{11}\right\|^{2}-\left\|\varrho_{02}\right\|^{2}+\Re\left(\bar{\lambda} \varrho_{21}\right),
$$


where

$$
\begin{aligned}
\varrho_{02}= & \left.\frac{1}{8}\left(\frac{\partial^{2} \bar{P}}{\partial P_{n}^{2}}-\frac{\partial^{2} \bar{P}}{\partial Z_{n}^{2}}+2 \frac{\partial^{2} \bar{Q}}{\partial P_{n} \partial Z_{n}}+\iota\left(\frac{\partial^{2} \bar{Q}}{\partial P_{n}^{2}}-\frac{\partial^{2} \bar{Q}}{\partial Z_{n}^{2}}+2 \frac{\partial^{2} \bar{P}}{\partial P_{n} \partial Z_{n}}\right)\right)\right|_{F_{00}(0,0)}, \\
\varrho_{11}= & \left.\frac{1}{4}\left(\frac{\partial^{2} \bar{P}}{\partial P_{n}^{2}}+\frac{\partial^{2} \bar{P}}{\partial Z_{n}^{2}}+\iota\left(\frac{\partial^{2} \bar{Q}}{\partial P_{n}^{2}}+\frac{\partial^{2} \bar{Q}}{\partial Z_{n}^{2}}\right)\right)\right|_{F_{00}(0,0)}, \\
\varrho_{20}= & \left.\frac{1}{8}\left(\frac{\partial^{2} \bar{P}}{\partial P_{n}^{2}}-\frac{\partial^{2} \bar{P}}{\partial Z_{n}^{2}}+2 \frac{\partial^{2} \bar{Q}}{\partial P_{n} \partial Z_{n}}+\iota\left(\frac{\partial^{2} \bar{Q}}{\partial P_{n}^{2}}-\frac{\partial^{2} \bar{Q}}{\partial Z_{n}^{2}}-2 \frac{\partial^{2} \bar{P}}{\partial P_{n} \partial Z_{n}}\right)\right)\right|_{F_{00}(0,0)}, \\
\varrho_{21}= & \frac{1}{16}\left(\frac{\partial^{3} \bar{P}}{\partial P_{n}^{3}}+\frac{\partial^{3} \bar{P}}{\partial Z_{n}^{3}}+\frac{\partial^{3} \bar{Q}}{\partial P_{n}^{2} \partial Z_{n}}+\frac{\partial^{3} \bar{Q}}{\partial Z_{n}^{3}}\right. \\
& \left.+\iota\left(\frac{\partial^{3} \bar{Q}}{\partial P_{n}^{3}}+\frac{\partial^{3} \bar{Q}}{\partial P_{n} \partial Z_{n}^{2}}-\frac{\partial^{3} \bar{P}}{\partial P_{n}^{2} \partial Z_{n}}-\frac{\partial^{3} \bar{P}}{\partial Z_{n}^{3}}\right)\right)\left.\right|_{F_{00}(0,0)} .
\end{aligned}
$$

After some manipulation, one gets

$$
\begin{aligned}
& \varrho_{02}=\frac{1}{4}\left(r_{12}+r_{13}+r_{24}+\iota\left(r_{22}+r_{23}+r_{14}\right)\right), \\
& \varrho_{11}=\frac{1}{4}\left(r_{12}+r_{13}+\iota\left(r_{22}+r_{23}\right)\right), \\
& \varrho_{20}=\frac{1}{4}\left(r_{12}+r_{13}+r_{24}+\iota\left(r_{22}+r_{23}-r_{14}\right)\right), \\
& \varrho_{21}=\frac{3}{8}\left(r_{11}+\iota r_{22}\right) .
\end{aligned}
$$

Finally, utilizing (71) into (69), if one gets $\Pi \neq 0$ as $\left.(\gamma, h, \beta, \omega, \nu) \in \mathcal{N}\right|_{F_{P Z}^{+}\left(\sqrt{\frac{\omega \nu^{2}}{1-\omega}}, \frac{\beta v\left(\sqrt{1-\omega}-\sqrt{\omega \nu^{2}}\right)}{\sqrt{\omega}(1-\omega)}\right)}$, then around $F_{P Z}^{+}\left(\sqrt{\frac{\omega \nu^{2}}{1-\omega}}, \frac{\beta \nu\left(\sqrt{1-\omega}-\sqrt{\omega \nu^{2}}\right)}{\sqrt{\omega}(1-\omega)}\right)$ phytoplankton-zooplankton model (8) undergoes the N-S bifurcation. Further supercritical (resp. subcritical) N-S bifurcation occurs if $\Pi<0$ (resp. $\Pi>0$ ).

\subsubsection{Flip bifurcation around $F_{P Z}^{+}\left(\sqrt{\frac{\omega \nu^{2}}{1-\omega}}, \frac{\beta v\left(\sqrt{1-\omega}-\sqrt{\omega \nu^{2}}\right)}{\sqrt{\omega}(1-\omega)}\right)$}

If (37) holds, then the eigenvalues of $\left.V\right|_{F_{P Z}^{+}\left(\sqrt{\frac{\omega \nu^{2}}{1-\omega}}, \frac{\beta v\left(\sqrt{1-\omega}-\sqrt{\omega \nu^{2}}\right)}{\sqrt{\omega}(1-\omega)}\right)}$ around $F_{P Z}^{+}\left(\sqrt{\frac{\omega \nu^{2}}{1-\omega}}\right.$, $\left.\frac{\beta \nu\left(\sqrt{1-\omega}-\sqrt{\omega \nu^{2}}\right)}{\sqrt{\omega}(1-\omega)}\right)$ satisfy $\left.\lambda_{2}\right|_{(37)}=-1$, but $\left.\lambda_{1}\right|_{(37)}=\frac{(3-h \beta) \sqrt{1-\omega}+2 h \beta \omega\left(\sqrt{1-\omega}-\sqrt{\omega \nu^{2}}\right)}{\sqrt{1-\omega}} \neq 1$ or -1 , which further shows the fact that around $F_{P Z}^{+}\left(\sqrt{\frac{\omega \nu^{2}}{1-\omega}}, \frac{\beta \nu\left(\sqrt{1-\omega}-\sqrt{\omega \nu^{2}}\right)}{\sqrt{\omega}(1-\omega)}\right)$ there may exist the flip bifurcation by choosing $\gamma$ as a bifurcation parameter if $(\gamma, h, \beta, \omega, \nu)$ are located in the set

$$
\begin{aligned}
& \mathcal{F}_{F_{P Z}^{+}\left(\sqrt{\frac{\omega \nu}{1-\omega}}, \frac{\beta \nu\left(\sqrt{1-\omega}-\sqrt{\omega \nu^{2}}\right)}{\sqrt{\omega(1-\omega)}}\right)} \\
& \quad:=\left\{(\gamma, h, \beta, \omega, \nu), \gamma=\frac{h \beta \sqrt{1-\omega}-2 \sqrt{1-\omega}-2 h \beta \omega\left(\sqrt{1-\omega}-\sqrt{\omega \nu^{2}}\right)}{h^{2} \beta \omega(1-\omega)\left(\sqrt{1-\omega}-\sqrt{\omega \nu^{2}}\right)}\right\} .
\end{aligned}
$$

But the following theorem guarantees the fact that around $F_{P Z}^{+}\left(\sqrt{\frac{\omega \nu^{2}}{1-\omega}}, \frac{\beta v\left(\sqrt{1-\omega}-\sqrt{\omega \nu^{2}}\right)}{\sqrt{\omega}(1-\omega)}\right)$ phytoplankton-zooplankton model (8) undergoes a flip bifurcation.

Theorem 5.4 If $\left.(\gamma, h, \beta, \omega, \nu) \in \mathcal{F}\right|_{F_{P Z}^{+}\left(\sqrt{\frac{\omega \nu^{2}}{1-\omega}}, \frac{\beta v\left(\sqrt{1-\omega}-\sqrt{\omega \nu^{2}}\right)}{\sqrt{\omega(1-\omega)}}\right)}$, then around $F_{P Z}^{+}\left(\sqrt{\frac{\omega \nu^{2}}{1-\omega}}\right.$, $\left.\frac{\beta \nu\left(\sqrt{1-\omega}-\sqrt{\omega \nu^{2}}\right)}{\sqrt{\omega}(1-\omega)}\right)$ phytoplankton-zooplankton model (8) undergoes a flip bifurcation. 
Proof Since $\left.(\gamma, h, \beta, \omega, v) \in \mathcal{F}\right|_{F_{P Z}^{+}\left(\sqrt{\frac{\omega \nu^{2}}{1-\omega},}, \frac{\beta v\left(\sqrt{1-\omega}-\sqrt{\omega \nu^{2}}\right)}{\sqrt{\omega(1-\omega)}}\right)}$, it is clear that $\gamma$ is the bifurcation parameter. Therefore, if $\gamma$ is a nbhd of $\gamma^{*}$, then phytoplankton-zooplankton model (8) takes the form which is depicted in (53). Further, phytoplankton-zooplankton model (8) takes the following form:

$$
\begin{aligned}
u_{n+1}= & \widehat{\alpha_{11}} u_{n}+\widehat{\alpha_{12}} v_{n}+\widehat{\alpha_{13}} u_{n}^{2}+\widehat{\alpha_{14}} u_{n} v_{n}+\widehat{\alpha_{15}} u_{n}^{3}+\widehat{\alpha_{16}} u_{n}^{2} v_{n}+\gamma_{01} u_{n} \epsilon \\
& +\gamma_{02} v_{n} \epsilon+\gamma_{03} u_{n}^{2} \epsilon+\gamma_{04} u_{n} v_{n} \epsilon+\gamma_{05} u_{n}^{3} \epsilon+\gamma_{06} u_{n}^{2} v_{n} \epsilon, \\
v_{n+1}= & \widehat{\alpha_{21}} u_{n}+\widehat{\alpha_{22}} v_{n}+\widehat{\alpha_{23}} u_{n}^{2}+\widehat{\alpha_{24}} u_{n} v_{n}+\widehat{\alpha_{25}} u_{n}^{3}+\widehat{\alpha_{26}} u_{n}^{2} v_{n}+\gamma_{07} u_{n} \epsilon \\
& +\gamma_{08} v_{n} \epsilon+\gamma_{09} u_{n}^{2} \epsilon+\gamma_{10} u_{n} v_{n} \epsilon+\gamma_{11} u_{n}^{3} \epsilon+\gamma_{12} u_{n}^{2} v_{n} \epsilon,
\end{aligned}
$$

where

$$
\begin{aligned}
& \widehat{\alpha_{11}}=1+h \beta-2 h \beta P^{*}-\frac{2 h v^{2} Z^{*} P *}{\left(v^{2}+P^{* 2}\right)^{2}}, \quad \widehat{\alpha_{12}}=-\frac{h P^{* 2}}{\left(v^{2}+P^{* 2}\right)^{2}}, \\
& \widehat{\alpha_{13}}=-h \beta-\frac{h Z^{*} v^{2}\left(v^{2}-3 P^{* 2}\right)}{\left(v^{2}+P^{* 2}\right)^{3}}, \quad \widehat{\alpha_{14}}=-\frac{2 h v^{2} P^{*}}{\left(v^{2}+P^{* 2}\right)^{2}}, \\
& \widehat{\alpha_{15}}=\frac{4 h Z^{*} v^{2} P^{*}\left(v^{2}-P^{* 2}\right)}{\left(v^{2}+P^{* 2}\right)^{4}}, \quad \widehat{\alpha_{16}}=-\frac{h v^{2}\left(v^{2}-3 P^{* 2}\right)}{\left(v^{2}+P^{* 2}\right)^{3}}, \\
& \gamma_{01}=\beta-2 \beta P-\frac{2 v^{2} Z^{*} P^{*}}{\left(v^{2}+P^{* 2}\right)^{2}}, \quad \gamma_{02}=-\frac{P^{* 2}}{\left(v^{2}+P^{* 2}\right)^{2}}, \\
& \gamma_{03}=-\beta-\frac{Z^{*} v^{2}\left(v^{2}-3 P^{* 2}\right)}{\left(v^{2}+P^{* 2}\right)^{3}}, \quad \gamma_{04}=-\frac{2 v^{2} P^{* 2}}{\left(v^{2}+P^{* 2}\right)^{2}}, \\
& \gamma_{05}=\frac{4 Z^{*} v^{2} P^{*}\left(v^{2}-P^{* 2}\right)}{\left(v^{2}+P^{* 2}\right)^{4}}, \quad \gamma_{06}=-\frac{v^{2}\left(v^{2}-3 P^{* 2}\right)}{\left(v^{2}+P^{* 2}\right)^{3}}, \\
& \widehat{\alpha_{21}}=\frac{2 h v^{2} \gamma^{*} Z^{*} P^{*}}{\left(v^{2}+P^{* 2}\right)^{2}}, \quad \widehat{\alpha_{22}}=1+h \gamma^{*} Z^{*}\left(\frac{P^{* 2}}{v^{2}+P^{* 2}}-\omega\right) \text {, } \\
& \widehat{\alpha_{23}}=\frac{h \gamma^{*} Z^{*} v^{2}\left(v^{2}-3 P^{* 2}\right)}{\left(v^{2}+P^{* 2}\right)^{3}}, \quad \widehat{\alpha_{24}}=\frac{2 h \gamma^{*} v^{2} P^{*}}{\left(v^{2}+P^{* 2}\right)^{2}}, \\
& \widehat{\alpha_{25}}=-\frac{4 h Z^{*} v^{2} \gamma^{*} P^{*}\left(v^{2}-P^{* 2}\right)}{\left(v^{2}+P^{* 2}\right)^{4}}, \quad \widehat{\alpha_{26}}=\frac{h v^{2} \gamma^{*}\left(v^{2}-3 P^{* 2}\right)}{\left(v^{2}+P^{* 2}\right)^{3}} \text {, } \\
& \gamma_{07}=\frac{2 v^{2} \gamma^{*} Z^{*} P^{*}}{\left(v^{2}+P^{* 2}\right)^{2}}, \quad \gamma_{08}=\gamma^{*} Z^{*}\left(\frac{P^{* 2}}{v^{2}+P^{* 2}}-\omega\right), \\
& \gamma_{09}=\frac{Z^{*} v^{2} \gamma^{*}\left(v^{2}-3 P^{* 2}\right)}{\left(v^{2}+P^{* 2}\right)^{3}}, \quad \gamma_{10}=\frac{2 \gamma^{*} v^{2} P^{*}}{\left(v^{2}+P^{* 2}\right)^{2}}, \\
& \gamma_{11}=-\frac{4 Z^{*} v^{2} \gamma^{*} P^{*}\left(v^{2}-P^{* 2}\right)}{\left(v^{2}+P^{* 2}\right)^{4}}, \quad \gamma_{12}=\frac{\gamma^{*} v^{2}\left(v^{2}-3 P^{* 2}\right)}{\left(v^{2}+P^{* 2}\right)^{3}}
\end{aligned}
$$

by using the transformation which is depicted in (59). Again it is noted that (73) takes the following form:

$$
\left(\begin{array}{c}
P_{n+1} \\
Z_{n+1}
\end{array}\right)=\left(\begin{array}{cc}
-1 & 0 \\
0 & \lambda_{1}
\end{array}\right)\left(\begin{array}{l}
P_{n} \\
Z_{n}
\end{array}\right)+\left(\begin{array}{l}
\widehat{P}\left(u_{n}, v_{n}, \epsilon\right) \\
\widehat{Q}\left(u_{n}, v_{n}, \epsilon\right)
\end{array}\right),
$$


where

$$
\begin{aligned}
& \widehat{P}\left(u_{n}, v_{n}, \epsilon\right)=\frac{\widehat{\alpha_{13}}\left(\lambda_{1}-\widehat{\alpha_{11}}\right)-\widehat{\alpha_{12}} \widehat{\alpha_{13}}}{\widehat{\alpha_{12}}\left(1+\lambda_{1}\right)} u_{n}^{2}+\frac{\widehat{\alpha_{14}}\left(\lambda_{1}-\widehat{\alpha_{11}}\right)-\widehat{\alpha_{12}} \widehat{\alpha_{24}}}{\widehat{\alpha_{12}}\left(1+\lambda_{1}\right)} u_{n} v_{n} \\
& +\frac{\widehat{\alpha_{15}}\left(\lambda_{1}-\widehat{\alpha_{11}}\right)-\widehat{\alpha_{12}} \widehat{\alpha_{25}}}{\widehat{\alpha_{12}}\left(1+\lambda_{1}\right)} u_{n}^{3}+\frac{\widehat{\alpha_{16}}\left(\lambda_{1}-\widehat{\alpha_{11}}\right)-\widehat{\alpha_{12}} \widehat{\alpha_{26}}}{\widehat{\alpha_{12}}\left(1+\lambda_{1}\right)} u_{n}^{2} v_{n} \\
& +\frac{\gamma_{01}\left(\lambda_{1}-\widehat{\alpha_{11}}\right)-\widehat{\alpha_{12}} \gamma_{07}}{\widehat{\alpha_{12}}\left(1+\lambda_{1}\right)} u_{n} \epsilon+\frac{\gamma_{02}\left(\lambda_{1}-\widehat{\alpha_{11}}\right)-\widehat{\alpha_{12}} \gamma_{08}}{\widehat{\alpha_{12}}\left(1+\lambda_{1}\right)} v_{n} \epsilon \\
& +\frac{\gamma_{03}\left(\lambda_{1}-\widehat{\alpha_{11}}\right)-\widehat{\alpha_{12}} \gamma_{09}}{\widehat{\alpha_{12}}\left(1+\lambda_{1}\right)} u_{n}^{2} \epsilon+\frac{\gamma 04\left(\lambda_{1}-\widehat{\alpha_{11}}\right)-\widehat{\alpha_{12}} \gamma_{10}}{\widehat{\alpha_{12}}\left(1+\lambda_{1}\right)} u_{n} v_{n} \epsilon \\
& +\frac{\gamma 05\left(\lambda_{1}-\widehat{\alpha_{11}}\right)-\widehat{\alpha_{12}} \gamma_{11}}{\widehat{\alpha_{12}}\left(1+\lambda_{1}\right)} u_{n}^{3} \epsilon+\frac{\gamma 06\left(\lambda_{1}-\widehat{\alpha_{11}}\right)-\widehat{\alpha_{12}} \gamma_{12}}{\widehat{\alpha_{12}}\left(1+\lambda_{1}\right)} u_{n}^{2} \nu_{n} \epsilon \text {, } \\
& \widehat{Q}\left(u_{n}, v_{n}, \epsilon\right)=\frac{\widehat{\alpha_{13}}\left(1+\widehat{\alpha_{11}}\right)+\widehat{\alpha_{12}} \widehat{\alpha_{13}}}{\widehat{\alpha_{12}}\left(1+\lambda_{1}\right)} u_{n}^{2}+\frac{\widehat{\alpha_{14}}\left(1+\widehat{\alpha_{11}}\right)+\widehat{\alpha_{12}} \widehat{\alpha_{24}}}{\widehat{\alpha_{12}}\left(1+\lambda_{1}\right)} u_{n} v_{n} \\
& +\frac{\widehat{\alpha_{15}}\left(1+\widehat{\alpha_{11}}\right)+\widehat{\alpha_{12}} \widehat{\alpha_{25}}}{\widehat{\alpha_{12}}\left(1+\lambda_{1}\right)} u_{n}^{3}+\frac{\widehat{\alpha_{16}}\left(1+\widehat{\alpha_{11}}\right)+\widehat{\alpha_{12}} \widehat{\alpha_{26}}}{\widehat{\alpha_{12}}\left(1+\lambda_{1}\right)} u_{n}^{2} v_{n} \\
& +\frac{\gamma_{01}\left(\lambda_{1}-\widehat{\alpha_{11}}\right)+\widehat{\alpha_{12}} \gamma_{07}}{\widehat{\alpha_{12}}\left(1+\lambda_{1}\right)} u_{n} \epsilon+\frac{\gamma_{02}\left(1+\widehat{\alpha_{11}}\right)+\widehat{\alpha_{12}} \gamma_{08}}{\widehat{\alpha_{12}}\left(1+\lambda_{1}\right)} v_{n} \epsilon \\
& +\frac{\gamma_{03}\left(1+\widehat{\alpha_{11}}\right)+\widehat{\alpha_{12}} \gamma_{09}}{\widehat{\alpha_{12}}\left(1+\lambda_{1}\right)} u_{n}^{2} \epsilon+\frac{\gamma 04\left(1+\widehat{\alpha_{11}}\right)+\widehat{\alpha_{12}} \gamma_{10}}{\widehat{\alpha_{12}}\left(1+\lambda_{1}\right)} u_{n} v_{n} \epsilon \\
& +\frac{\gamma 05\left(1+\widehat{\alpha_{11}}\right)+\widehat{\alpha_{12}} \gamma_{11}}{\widehat{\alpha_{12}}\left(1+\lambda_{1}\right)} u_{n}^{3} \epsilon+\frac{\gamma 06\left(1+\widehat{\alpha_{11}}\right)+\widehat{\alpha_{12}} \gamma_{12}}{\widehat{\alpha_{12}}\left(1+\lambda_{1}\right)} u_{n}^{2} v_{n} \epsilon \text {, } \\
& u_{n}=\widehat{\alpha_{12}} P_{n}+\widehat{\alpha_{12}} Z_{n} \text {, } \\
& v_{n}=-\left(1+\widehat{\alpha_{11}}\right) P_{n}+\left(\lambda_{1}-\widehat{\alpha_{11}}\right) Z_{n} \text {, } \\
& u_{n}^{2}={\widehat{\alpha_{12}}}^{2}\left(P_{n}^{2}+2 P_{n} Z_{n}+Z_{n}^{2}\right) \text {, } \\
& v_{n}^{2}=\left(1+\widehat{\alpha_{11}}\right)^{2} P_{n}^{2}+\left(\lambda_{1}-\widehat{\alpha_{11}}\right)^{2} Z_{n}^{2}-2\left(1+\widehat{\alpha_{11}}\right)\left(\lambda_{1}-\widehat{\alpha_{11}}\right) P_{n} Z_{n} \text {, } \\
& u_{n}^{3}={\widehat{\alpha_{12}}}^{3}\left[P_{n}^{3}+Z_{n}^{3}+3 P_{n}^{2} Z_{n}+3 P_{n} Z_{n}^{2}\right] \text {, } \\
& u_{n} v_{n}=-\widehat{\alpha_{12}}\left(1+\widehat{\alpha_{11}}\right) P_{n}^{2}+\left(\widehat{\alpha_{12}}\left(\lambda_{1}-\widehat{\alpha_{11}}\right)-\widehat{\alpha_{12}}\left(1+\widehat{\alpha_{11}}\right)\right) P_{n} Z_{n} \widehat{\alpha_{12}}\left(\lambda_{1}-\widehat{\alpha_{11}}\right) Z_{n}^{2} \text {, } \\
& u_{n}^{2} v_{n}=-{\widehat{\alpha_{12}}}^{2}\left[\left(1+\widehat{\alpha_{11}}\right)^{3}+P_{n}^{2} Z_{n}\left(2+\widehat{\alpha_{11}}+\lambda_{1}\right)+\left(1-\widehat{\alpha_{11}}+2 \lambda_{1}\right) P_{n} Z_{n}^{2}\right. \\
& \left.+\left(\lambda_{1}-\widehat{\alpha_{11}}\right)^{3} Z_{n}^{3}\right] \text {, } \\
& u_{n} \epsilon=\widehat{\alpha_{12}} P_{n} \epsilon+\widehat{\alpha_{12}} Z_{n} \epsilon \text {, } \\
& v_{n} \epsilon=-\left(1+\widehat{\alpha_{11}}\right) P_{n} \epsilon+\left(\lambda_{1}-\widehat{\alpha_{11}}\right) Z_{n} \epsilon \text {, } \\
& u_{n}^{2} \epsilon=\epsilon{\widehat{\alpha_{12}}}^{2}\left(P_{n}^{2}+2 P_{n} Z_{n}+Z_{n}^{2}\right) \text {, } \\
& v_{n}^{2} \epsilon=\left(1+\widehat{\alpha_{11}}\right)^{2} P_{n}^{2} \epsilon+\left(\lambda_{1}-\widehat{\alpha_{11}}\right)^{2} Z_{n}^{2} \epsilon-2\left(1+\widehat{\alpha_{11}}\right)\left(\lambda_{1}-\widehat{\alpha_{11}}\right) P_{n} Z_{n} \epsilon \text {, } \\
& u_{n}^{3} \epsilon={\widehat{\alpha_{12}}}^{3} \epsilon\left[P_{n}^{3}+Z_{n}^{3}+3 P_{n}^{2} Z_{n}+3 P_{n} Z_{n}^{2}\right] \text {, } \\
& \left.u_{n} v_{n} \epsilon=-\widehat{\alpha_{12}}\left(1+\widehat{\alpha_{11}}\right) P_{n}^{2} \epsilon+\widehat{\left(\alpha_{12}\right.}\left(\lambda_{1}-\widehat{\alpha_{11}}\right)-\widehat{\alpha_{12}}\left(1+\widehat{\alpha_{11}}\right)\right) P_{n} Z_{n} \epsilon \\
& +\widehat{\alpha_{12}}\left(\lambda_{1}-\widehat{\alpha_{11}}\right) Z_{n}^{2} \epsilon \text {, } \\
& u_{n}^{2} v_{n} \epsilon=-{\widehat{\alpha_{12}}}^{2}\left[\left(1+\widehat{\alpha_{11}}\right)^{3} \epsilon+P_{n}^{2} Z_{n}\left(2+\widehat{\alpha_{11}}+\lambda_{1}\right) \epsilon+\left(1-\widehat{\alpha_{11}}+2 \lambda_{1}\right) P_{n} Z_{n}^{2} \epsilon\right. \\
& \left.+\left(\lambda_{1}-\widehat{\alpha_{11}}\right)^{3} Z_{n}^{3} \epsilon\right] \text {, }
\end{aligned}
$$


by

$$
\left(\begin{array}{l}
u_{n} \\
v_{n}
\end{array}\right)=\left(\begin{array}{cc}
\widehat{\alpha_{12}} & \widehat{\alpha_{12}} \\
-1-\widehat{\alpha_{11}} & \lambda_{1}-\widehat{\alpha_{11}}
\end{array}\right)\left(\begin{array}{l}
P_{n} \\
Z_{n}
\end{array}\right) .
$$

Hereafter for $(75)$ the center manifold $M^{c} F_{00}(0,0)$ around $F_{00}(0,0)$ is investigated in a small nbhd of $\epsilon$, and hence one can write $M^{c} F_{00}(0,0)$ as the following mathematical expression:

$$
M^{c} F_{00}(0,0)=\left\{\left(P_{n}, Z_{n}\right): Z_{n}=C_{0} \epsilon+C_{1} P_{n}^{2}+C_{2} P_{n} \epsilon+C_{3} \epsilon^{3}+O\left(\left(\left|P_{n}\right|+|\epsilon|\right)^{3}\right)\right\} .
$$

The computation yields

$$
\begin{aligned}
& C_{0}=0, \\
& C_{1}=\frac{\left(1+\widehat{\alpha_{11}}\right)}{1-\lambda_{1}^{2}}\left(\widehat{\alpha_{12}} \widehat{\alpha_{13}}-\left(1+\widehat{\alpha_{11}}\right) \widehat{\alpha_{14}}-\widehat{\alpha_{12}} \widehat{\alpha_{24}}\right)+\frac{1}{1-\lambda_{1}^{2}}{\widehat{\alpha_{12}}}^{2} \widehat{\alpha_{23}}, \\
& C_{2}=\frac{\left(\gamma_{01}+\gamma_{08}\right)\left(1+\widehat{\alpha_{11}}\right) \widehat{\alpha_{12}}+\gamma_{07}{\widehat{\alpha_{12}}}^{2}-\gamma_{02}\left(1+\widehat{\alpha_{11}}\right)^{2}}{C_{3}}, \quad 0 .
\end{aligned}
$$

Finally, one can express (75) restricted to $M^{c} F_{00}(0,0)$ as follows:

$$
f\left(P_{n}\right)=-P_{n}+h_{1} P_{n}^{2}+h_{2} P_{n} \epsilon+h_{3} P_{n}^{2} \epsilon+h_{4} P_{n} \epsilon^{2}+h_{5} P_{n}^{3}+O\left(\left(\left|P_{n}\right|+|\epsilon|\right)^{4}\right),
$$

where

$$
\begin{aligned}
& h_{1}=\frac{1}{1+\lambda_{1}}\left[\widehat{\alpha_{12}} \widehat{\alpha_{13}}\left(\lambda_{1}-\widehat{\alpha_{11}}\right)-\left(1+\widehat{\alpha_{11}}\right)\left(\widehat{\alpha_{14}}\left(\lambda_{1}-\widehat{\alpha_{11}}\right)-\widehat{\alpha_{12}} \widehat{\alpha_{24}}\right)-{\widehat{\alpha_{12}}}^{2} \widehat{\alpha_{23}}\right] \text {, } \\
& h_{2}=\frac{1}{\left(1+\lambda_{1}\right)}\left[\left(\gamma_{01}\left(\lambda_{1}-\widehat{\alpha_{11}}\right)-\gamma_{07} \widehat{\alpha_{12}}\right)-\frac{\left(1+\widehat{\alpha_{11}}\right)\left(\gamma_{02}\left(\lambda_{1}-\widehat{\alpha_{11}}\right)-\widehat{\alpha_{12} \gamma_{8}}\right)}{\widehat{\alpha_{12}}}\right] \text {, } \\
& h_{3}=\frac{1}{\widehat{\alpha_{12}}\left(1+\lambda_{1}\right)}\left[2 C_{2}{\widehat{\alpha_{12}}}^{2}\left(\widehat{\alpha_{13}}\left(\lambda_{1}-\widehat{\alpha_{11}}\right)-\widehat{\alpha_{12}} \widehat{\alpha_{23}}\right)\right. \\
& +C_{2} \widehat{\alpha_{12}}\left(\left(\lambda_{1}-\widehat{\alpha_{11}}\right)-\left(1+\widehat{\alpha_{11}}\right)\right)\left(\widehat{\alpha_{14}}\left(\lambda_{1}-\widehat{\alpha_{11}}\right)\right) \\
& -\widehat{\alpha_{12}} \widehat{\alpha_{24}}+\left(\gamma_{01}\left(\lambda_{1}-\widehat{\alpha_{11}}\right)-\widehat{\alpha_{12}} \gamma_{07}\right) C_{1} \widehat{\alpha_{12}} \\
& +C_{1}\left(\lambda_{1}-\widehat{\alpha_{11}}\right)\left(\gamma_{02}\left(\lambda_{1}-\widehat{\alpha_{11}}\right)-\widehat{\alpha_{12}} \gamma_{08}\right) \\
& +\left(\gamma_{03}\left(\lambda_{1}-\widehat{\alpha_{11}}\right)-\widehat{\alpha_{12}} \gamma_{09}\right){\widehat{\alpha_{12}}}^{2} \\
& \left.-\widehat{\alpha_{12}}\left(1+\widehat{\alpha_{11}}\right)\left(\gamma_{04}\left(\lambda_{1}-\widehat{\alpha_{11}}\right)-\widehat{\alpha_{12}} \gamma_{10}\right)\right] \\
& h_{4}=\frac{C_{2}}{\left(1+\lambda_{1}\right) \widehat{\alpha_{12}}}\left[\left(\gamma_{01}\left(\lambda_{1}-\widehat{\alpha_{11}}\right)-\widehat{\alpha_{12}} \gamma_{07}\right)+\left(\lambda_{1}-\widehat{\alpha_{11}}\right)\left(\gamma_{02}\left(\lambda_{1}-\widehat{\alpha_{11}}\right)-\widehat{\alpha_{12}} \gamma_{08}\right)\right] \text {, } \\
& h_{5}=\frac{1}{\left(1+\lambda_{1}\right)}\left[2 C_{1} \widehat{\alpha_{12}}\left(\widehat{\alpha_{13}}\left(\lambda_{1}-\widehat{\alpha_{11}}\right)-\widehat{\alpha_{12}} \widehat{\alpha_{23}}\right)\right. \\
& +C_{1}\left(\left(\lambda_{1}-\widehat{\alpha_{11}}\right)-\left(1+\widehat{\alpha_{11}}\right)\right)\left(\widehat{\alpha_{14}}\left(\lambda_{1}-\widehat{\alpha_{11}}\right)-\widehat{\alpha_{12}} \widehat{\alpha_{24}}\right) \\
& \left.+\widehat{\alpha_{12}^{2}}\left(\widehat{\alpha_{15}}\left(\lambda_{1}-\widehat{\alpha_{11}}\right)-\widehat{\alpha_{12}} \widehat{\alpha_{25}}\right)-\widehat{\alpha_{12}}\left(1+\widehat{\alpha_{11}}\right)\left(\widehat{\alpha_{16}}\left(\lambda_{1}-\widehat{\alpha_{11}}\right)-\widehat{\alpha_{12}} \widehat{\alpha_{26}}\right)\right] \text {. }
\end{aligned}
$$


Now it is required that the following discriminatory quantities are nonzero for map (80) to undergo the flip bifurcation $[8,9]$

$$
\begin{aligned}
& \Gamma_{1}=\left.\left(\frac{\partial^{2} f}{\partial P_{n} \partial \epsilon}+\frac{1}{2} \frac{\partial f}{\partial \epsilon} \frac{\partial^{2} f}{\partial P_{n}^{2}}\right)\right|_{F_{00}(0,0)}, \\
& \Gamma_{2}=\left.\left(\frac{1}{6} \frac{\partial^{3} f}{\partial P_{n}^{3}}+\left(\frac{1}{2} \frac{\partial^{2} f}{\partial P_{n}^{2}}\right)^{2}\right)\right|_{F_{00}(0,0)} .
\end{aligned}
$$

Therefore after computation we get

$$
\Gamma_{1}=-\frac{1}{h^{*}} \neq 0
$$

and

$$
\begin{aligned}
& \Gamma_{2}=\frac{\sqrt{1-\omega}}{(2-h \beta) \sqrt{1-\omega}+2 h \beta \omega\left(\sqrt{1-\omega}-\sqrt{\omega \nu^{2}}\right)} \\
& \times\left[\frac{-\sqrt{1-\omega}}{(4-h \beta) \sqrt{1-\omega}+2 h \beta \omega\left(\sqrt{1-\omega}-\sqrt{\omega \nu^{2}}\right)}\right. \\
& \times\left[\frac{-h^{2} \beta \omega\left(\sqrt{1-\omega}-\sqrt{\omega \nu^{2}}\right)(1-4 \omega)(1-\omega)}{\sqrt{\omega v^{2}}}\right. \\
& -\frac{(2-h \beta) \sqrt{1-\omega}+4 h^{2} \beta \omega^{2}\left(\sqrt{1-\omega}-\sqrt{\omega \nu^{2}}\right)(1-\omega)}{\sqrt{1-\omega}} \\
& +\frac{h \omega\left(\sqrt{1-\omega}-2 \omega\left(\sqrt{1-\omega}-\sqrt{\omega \nu^{2}}\right)\right)}{\sqrt{1-\omega}-\sqrt{\omega \nu^{2}}} \\
& -\frac{\sqrt{1-\omega}}{(2-h \beta) \sqrt{1-\omega}+2 h \beta \omega\left(\sqrt{1-\omega}-\sqrt{\omega \nu^{2}}\right)} \\
& \left.\times\left(\frac{h^{3} \omega^{2} \beta\left(\sqrt{1-\omega}-\sqrt{\omega \nu^{2}}\right)(1-4 \omega)(1-\omega)}{\sqrt{\omega \nu^{2}}}\right)\right] \\
& -2 h \omega\left(-h \beta\left(1+\left(\sqrt{1-\omega}-\sqrt{\omega \nu^{2}}\right)\right)(1-\omega)(1-4 \omega)\right. \\
& \left.+\frac{h^{2} \omega \beta\left(\sqrt{1-\omega}-\sqrt{\omega \nu^{2}}\right)(1-4 \omega)(1-\omega)}{\sqrt{\omega \nu^{2}}}\right) \\
& +\left(\frac{h \beta \sqrt{1-\omega}+2 h \beta \omega\left(\sqrt{1-\omega}-\sqrt{\omega \nu^{2}}\right)}{\sqrt{1-\omega}}\right) \\
& \times\left(-4 h \omega(1-\omega)+\frac{h \omega\left(\sqrt{1-\omega}-2 \omega\left(\sqrt{1-\omega}-\sqrt{\omega \nu^{2}}\right)\right)}{\sqrt{1-\omega}-\sqrt{\omega \nu^{2}}}\right) \\
& +h^{2} \omega^{2}\left(\frac{8 h \beta\left(\sqrt{1-\omega}-\sqrt{\omega \nu^{2}}\right)(1-2 \omega)(1-\omega)^{2}}{v^{2} \sqrt{1-\omega}}\right. \\
& \left.-\frac{2 h \beta \omega\left(\sqrt{1-\omega}-2 \omega\left(\sqrt{1-\omega}-\sqrt{\omega \nu^{2}}\right)\right)(1-2 \omega)(1-\omega)}{\nu^{2} \omega}\right) \\
& +\frac{h \omega(2-h \beta) \sqrt{1-\omega}+2 h \beta \omega\left(\sqrt{1-\omega}-\sqrt{\omega \nu^{2}}\right)}{\sqrt{1-\omega}}\left(\frac{-2 h(1-4 \omega)(1-\omega)^{2}}{\nu^{2}}\right.
\end{aligned}
$$




$$
\begin{aligned}
& \left.\left.+h \omega \frac{\left(\sqrt{1-\omega}-2 \omega\left(\sqrt{1-\omega}-\sqrt{\omega \nu^{2}}\right)\right)(1-4 \omega)(1-\omega)}{2 \omega\left(\sqrt{1-\omega}-\sqrt{\omega \nu^{2}}\right) \nu^{2}}\right)\right] \\
& +\left[\left(\frac{\sqrt{1-\omega}}{(4-h \beta) \sqrt{1-\omega}+2 h \beta \omega\left(\sqrt{1-\omega}-\sqrt{\omega \nu^{2}}\right)}\right)\right. \\
& \times\left(2 h^{2} \beta \omega\left(1+\left(\sqrt{1-\omega}-\sqrt{\omega \nu^{2}}\right)\right)(1-\omega)(1-4 \omega)\right. \\
& -\frac{h^{3} \omega^{2} \beta\left(\sqrt{1-\omega}-\sqrt{\omega \nu^{2}}\right)(1-\omega)(1-4 \omega)}{\sqrt{\omega \nu^{2}}} \\
& -\frac{(2-h \beta) \sqrt{1-\omega}+2 h \beta \omega\left(\sqrt{1-\omega}-\sqrt{\omega \nu^{2}}\right)}{\sqrt{1-\omega}} \\
& \left.\times\left(-4 h \omega(1-\omega)+\frac{h \omega\left(\sqrt{1-\omega}-2 \omega\left(\sqrt{1-\omega}-\sqrt{\omega \nu^{2}}\right)\right)}{\sqrt{1-\omega}-\sqrt{\omega \nu^{2}}}\right)\right]^{2} .
\end{aligned}
$$

Finally, from (84) if $\Gamma_{2} \neq 0$ as $\left.(\gamma, h, \beta, \omega, \nu) \in \mathcal{F}\right|_{F_{P Z}^{+}\left(\sqrt{\frac{\omega \nu^{2}}{1-\omega}}, \frac{\beta \nu\left(\sqrt{1-\omega}-\sqrt{\omega \nu^{2}}\right)}{\sqrt{\omega}(1-\omega)}\right)}$, then around the interior fixed point $F_{P Z}^{+}\left(\sqrt{\frac{\omega \nu^{2}}{1-\omega}}, \frac{\beta \nu\left(\sqrt{1-\omega}-\sqrt{\omega \nu^{2}}\right)}{\sqrt{\omega}(1-\omega)}\right)$, phytoplankton-zooplankton model (8) undergoes the flip bifurcation. Further, if $\Gamma_{2}>0$ (respectively $\Gamma_{2}<0$ ), then period-2 points bifurcating from $F_{P Z}^{+}\left(\sqrt{\frac{\omega \nu^{2}}{1-\omega}}, \frac{\beta \nu\left(\sqrt{1-\omega}-\sqrt{\omega \nu^{2}}\right)}{\sqrt{\omega}(1-\omega)}\right)$ are stable (respectively unstable).

\section{Chaos control}

In this section, the state feedback control method is utilized to stabilize chaos at the state of unstable trajectories by adding $u_{n}$ as control forces to discrete-time phytoplanktonzooplankton model $[22,23]$ :

$$
\begin{aligned}
& P_{n+1}=(1+h \beta) P_{n}-h\left(\beta+\frac{Z_{n}}{v^{2}+P_{n}^{2}}\right) P_{n}^{2}+u_{n} \\
& Z_{n+1}=Z_{n}+h \gamma Z_{n}\left(\frac{P_{n}^{2}}{v^{2}+P_{n}^{2}}-\omega\right) \\
& u_{n}=-k_{1}\left(P_{n}-P\right)-k_{2}\left(Z_{n}-Z\right)
\end{aligned}
$$

where $k_{1}, k_{2}$ denote feedback gains, and $P=\sqrt{\frac{\omega \nu^{2}}{1-\omega}}, Z=\frac{\beta \nu\left(\sqrt{1-\omega}-\sqrt{\omega \nu^{2}}\right)}{\sqrt{\omega}(1-\omega)}$. Now, for control system (85), the variational matrix $\left.V C\right|_{P_{P Z}(P, Z)}$ takes the following form:

$$
\left.V C\right|_{P_{P Z}(P, Z)}=\left(\begin{array}{cc}
\ell_{11}-k_{1} & \ell_{12}-k_{2} \\
\ell_{21} & \ell_{22}
\end{array}\right),
$$

where

$$
\begin{aligned}
& \ell_{11}=\frac{\sqrt{1-\omega}(1-h \beta)+2 h \beta \omega\left(\sqrt{1-\omega}-\sqrt{\omega \nu^{2}}\right)}{\sqrt{1-\omega}}, \\
& \ell_{12}=-h \omega, \\
& \ell_{21}=\frac{\beta\left(\sqrt{1-\omega}-2 \omega\left(\sqrt{1-\omega}-\sqrt{\omega \nu^{2}}\right)\right)}{\omega \sqrt{1-\omega}} . \\
& \ell_{22}=1 .
\end{aligned}
$$


Now, if the roots of the characteristic equation of $\left.V C\right|_{P_{P Z}(P, Z)}$ are $\lambda_{1,2}$, then

$$
\begin{aligned}
& \lambda_{1}+\lambda_{2}=\ell_{11}+\ell_{22}-k_{1}, \\
& \lambda_{1} \lambda_{2}=\ell_{22}\left(\ell_{11}-k_{1}\right)-\ell_{21}\left(\ell_{12}-k_{2}\right) .
\end{aligned}
$$

Now it is noted here that marginal stability is determined from the restrictions $\lambda_{1}= \pm 1$, $\lambda_{1} \lambda_{2}=1$, which gives the fact that $\left|\lambda_{1,2}\right|<1$. If $\lambda_{1} \lambda_{2}=1$, then from (89) one gets

$$
L_{1}: k_{1}-k_{2} \beta \frac{\left(\sqrt{1-\omega}-2 \omega\left(\sqrt{1-\omega}-\sqrt{\omega \nu^{2}}\right)\right)}{\omega \sqrt{1-\omega}}=0
$$

If $\lambda_{1}=1$, then from (88) and (89) one gets

$$
L_{2}: k_{2}+h \omega=0 \text {. }
$$

Finally, if $\lambda_{1}=-1$, then from (88) and (89) one gets

$$
\begin{aligned}
& L_{3}: 2 k_{1}-\beta\left(\frac{\sqrt{1-\omega}-2 \omega\left(\sqrt{1-\omega}-\sqrt{\omega \nu^{2}}\right)}{\omega \sqrt{1-\omega}}\right) k_{2} \\
&-\frac{(4-h \beta) \sqrt{1-\omega}+2 \omega h \beta\left(\sqrt{1-\omega}-\sqrt{\omega \nu^{2}}\right)}{\sqrt{1-\omega}}=0 .
\end{aligned}
$$

Therefore, from (90), (91), and (92), lines $L_{1}, L_{2}$, and $L_{3}$ in the ( $\left.k_{1}, k_{2}\right)$-plane give the triangular region, which further gives the fact that $\left|\lambda_{1,2}\right|<1$.

\section{Numerical simulations}

The obtained results are numerically verified in this section for fixing suitable values of involved parameters. Here the following two cases are considered for the completeness of this section.

Case 1: Let $h=0.95, \beta=0.55, v=0.6, \omega=0.23$, then from (33) one gets $\gamma=$ 3.0548345625166413 , which is the value of a bifurcation parameter. So, the interior fixed point $F_{P Z}^{+}\left(\sqrt{\frac{\omega \nu^{2}}{1-\omega}}, \frac{\beta \nu\left(\sqrt{1-\omega}-\sqrt{\omega \nu^{2}}\right)}{\sqrt{\omega}(1-\omega)}\right)$ of phytoplankton-zooplankton model (8) is a stable (respectively unstable) focus if $0<\gamma<3.0548345625166413$ (respectively $\gamma>$ 3.0548345625166413). To show this fact deeply, if one chooses the bifurcation value $\gamma=1.7<3.0548345625166413$, then Fig. 1 (a) shows that $F_{P Z}^{+}(0.3279214350000127$, 0.5270170961180427) of the discrete phytoplankton-zooplankton model (8) is a stable focus; moreover, Fig. 1(b)-1(f) shows the same qualitative behavior if bifurcation values respectively are $\gamma=1.97,2.1,2.35,3.01,3.01345<3.0548345625166413$. On the other hand, if $\gamma=3.3>3.0548345625166413$, then Fig. 2(a) shows that the positive fixed point $F_{P Z}^{+}\left(\sqrt{\frac{\omega \nu^{2}}{1-\omega}}, \frac{\beta \nu\left(\sqrt{1-\omega}-\sqrt{\omega \nu^{2}}\right)}{\sqrt{\omega}(1-\omega)}\right)$ of phytoplankton-zooplankton model (8) changes behavior, and as a consequence an attracting invariant closed curve appears. Now numerically we have to show that if $\gamma=3.3>3.0548345625166413$, then phytoplankton-zooplankton model (8) undergoes the supercritical N-S bifurcation, that is, the discriminatory quantity $\Pi<0$. So, if $\gamma=3.3$, then from (57) one gets $\left.\frac{d\left|\lambda_{1,2}\right|}{d \epsilon}\right|_{\epsilon=0}=0.7399820615275017>0$, i.e., nondegenerate condition for the existence of N-S bifurcation holds. Moreover, from (54) and (71) 


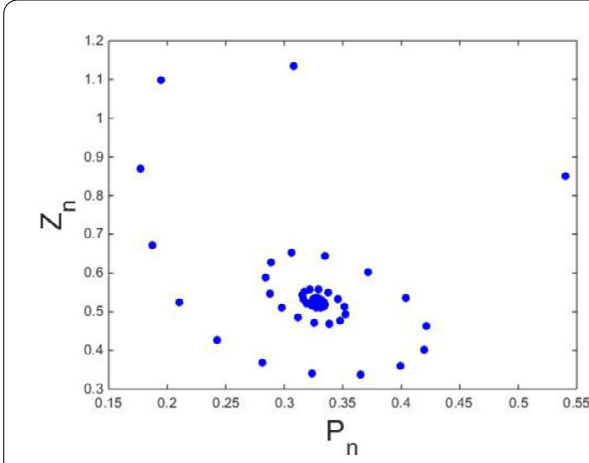

(a) $\gamma=1.7$ with $(0.54,0.85)$

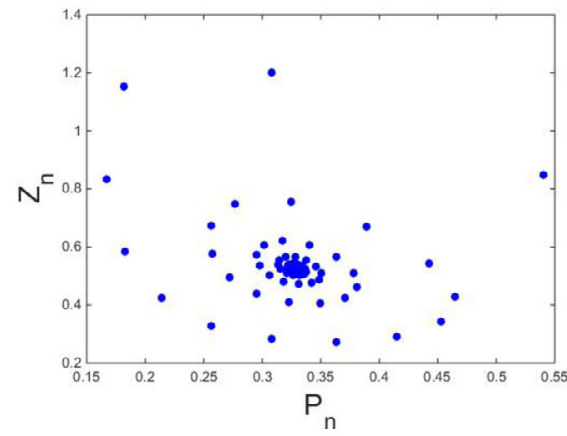

(c) $\gamma=2.1$ with $(0.54,0.85)$

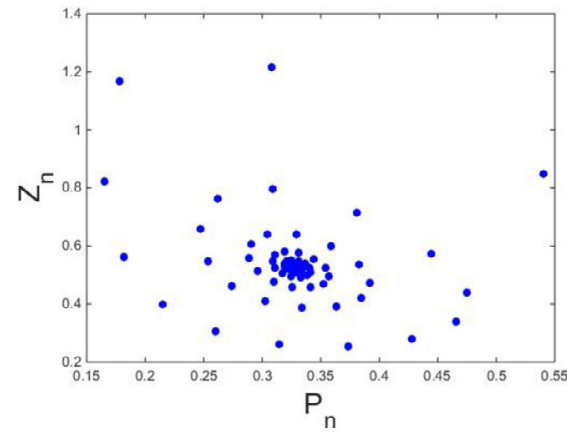

(e) $\gamma=3.01$ with $(0.54,0.85)$

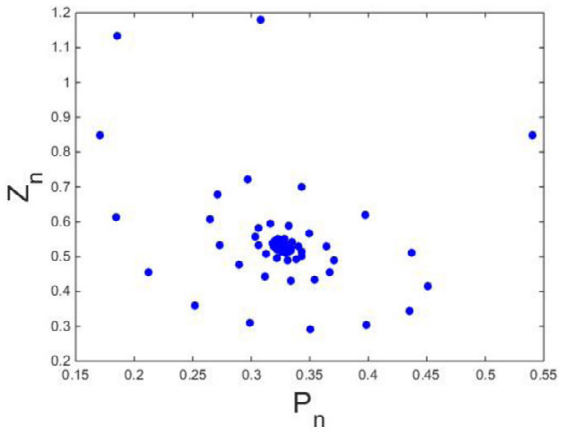

(b) $\gamma=1.97$ with $(0.54,0.85)$

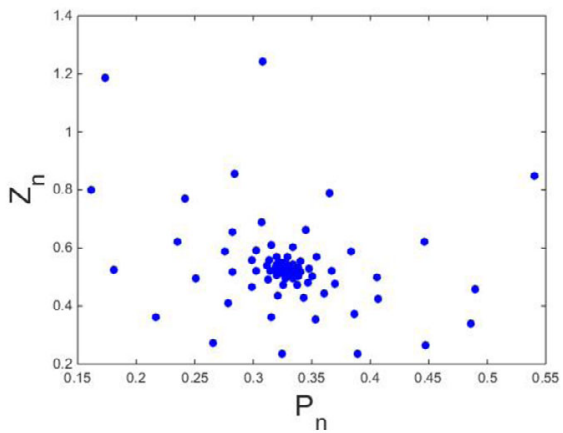

(d) $\gamma=2.35$ with $(0.54,0.85)$

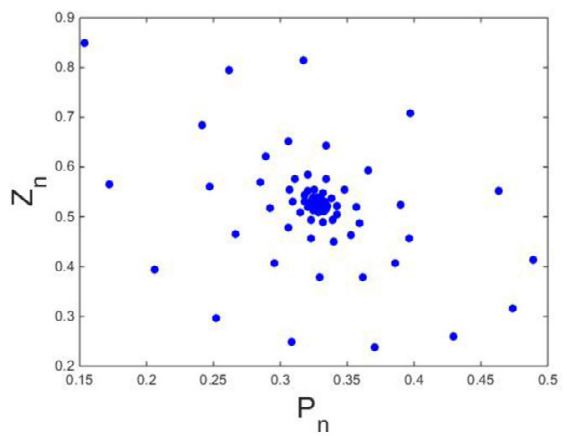

(f) $\gamma=3.01345$ with $(0.54,0.85)$

Figure 1 Stable focus of phytoplankton-zooplankton model (8)

one gets

$$
\lambda_{1,2}=0.8195170415488735 \pm 1.2288296165874808 \iota
$$

and

$$
\begin{aligned}
& \varrho_{02}=-0.1064845211021958+0.3409285950222708 \iota, \\
& \varrho_{11}=-0.022556674629067297+0.0424976106628709 \iota, \\
& \varrho_{20}=-0.022556674629067297+0.0424976106628709 \iota, \\
& \varrho_{21}=0.008072080753930323+0.022202436334813922 \iota .
\end{aligned}
$$




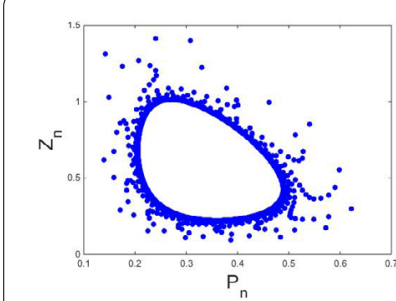

(a) $\gamma=3.3$ with $(0.54,0.85)$

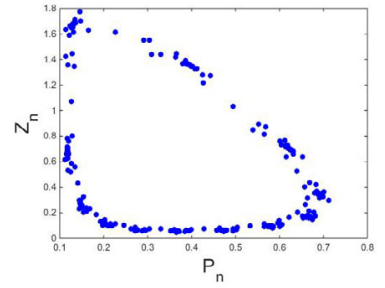

(d) $\gamma=3.54$ with $(0.54,0.85)$

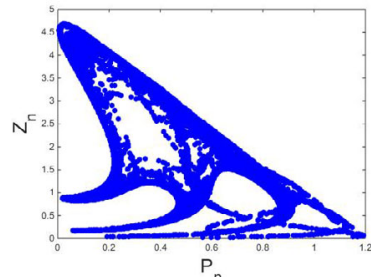

(g) $\gamma=3.87$ with $(0.0001,0.00001)$

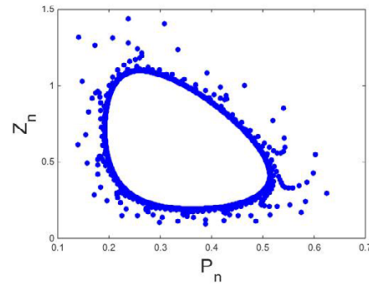

(b) $\gamma=3.324$ with $(0.54,0.85)$

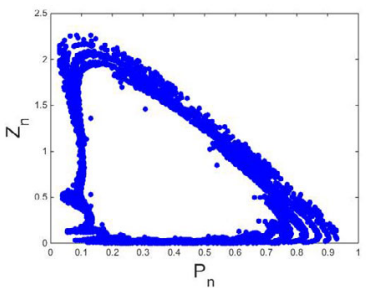

(e) $\gamma=3.654$ with $(0.54,0.85)$

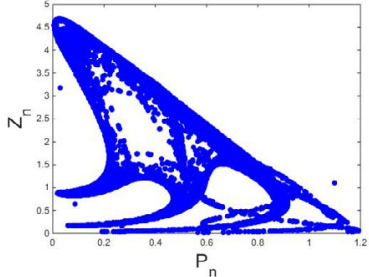

(h) $\gamma=3.9$ with $(0.0001,0.00001)$

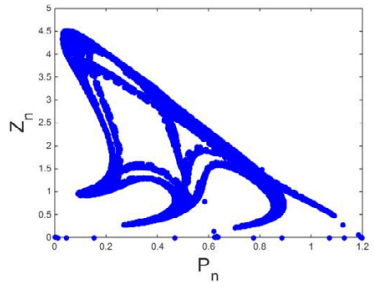

(j) $\gamma=4.95$ with $(0.0001,0.00001)$

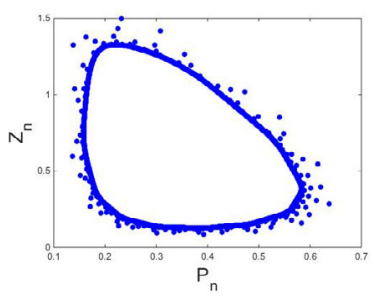

(c) $\gamma=3.4$ with $(0.54,0.85)$

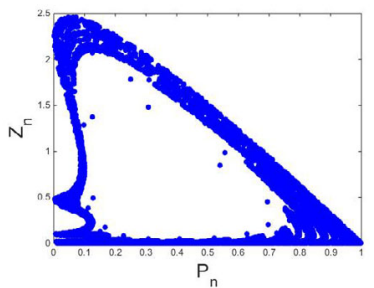

(f) $\gamma=3.8$ with $(0.54,0.85)$

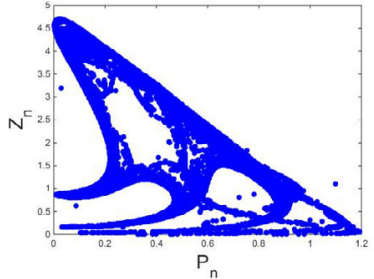

(i) $\gamma=4.22$ with $(1.1,1.1)$

Figure 2 Attracting invariant closed curves of phytoplankton-zooplankton model (8)

Using (93) and (94) in (69), one gets $\Pi=-0.11850669853573864<0$, which confirms that our obtained theoretical results are mathematically correct, and hence phytoplanktonzooplankton model (8) undergoes a supercritical N-S bifurcation. Similarly, for other chosen bifurcations values $\gamma=3.324,3.4,3.54,3.654,3.8,3.87,3.9,4.22,4.95>$ 3.0548345625166413 it is also from Fig. 2(b) $-2(j)$ that attracting invariant closed curves appear, and therefore the discrete phytoplankton-zooplankton model (8) undergoes a supercritical N-S bifurcation, i.e., for the said bifurcation values, $\Pi<0$ (see Table 1). Finally, bifurcation diagrams along with maximum Lyapunov exponent are drawn and presented in Fig. 3.

Case 2: If $h=0.9, \beta=3.7, v=0.6, \omega=0.523$, then from (37) one gets $\gamma=$ 0.12657824922905964 . From the theoretical discussion, the interior fixed point $F_{P Z}^{+}\left(\sqrt{\frac{\omega \nu^{2}}{1-\omega}}\right.$, $\left.\frac{\beta \nu\left(\sqrt{1-\omega}-\sqrt{\omega \nu^{2}}\right)}{\sqrt{\omega}(1-\omega)}\right)$ of the discrete phytoplankton-zooplankton model (8) is a stable (respec- 
Table 1 Numerical values of $\Pi$ for $\gamma>3.0548345625166413$

\begin{tabular}{ll}
\hline Bifurcation values if $\gamma>3.0548345625166413$ & Corresponding value of $\Pi$ \\
\hline 3.3 & $-0.11850669853573864<0$ \\
3.324 & $-0.11850669853573864<0$ \\
3.4 & $-0.11850669853573864<0$ \\
3.54 & $-0.13479326654465815<0$ \\
3.654 & $-0.14288926265451063<0$ \\
3.8 & $-0.15359841432110483<0$ \\
3.87 & $-0.1588691528795736<0$ \\
3.9 & $-0.16115514866851807<0$ \\
4.22 & $-0.18655628927087037<0$ \\
4.95 & $-0.2387294887287743<0$ \\
\hline
\end{tabular}

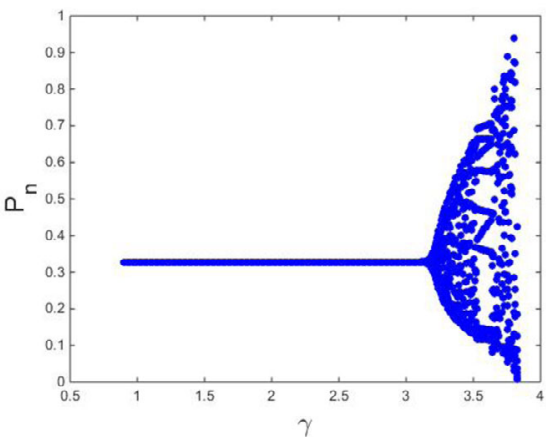

(a)

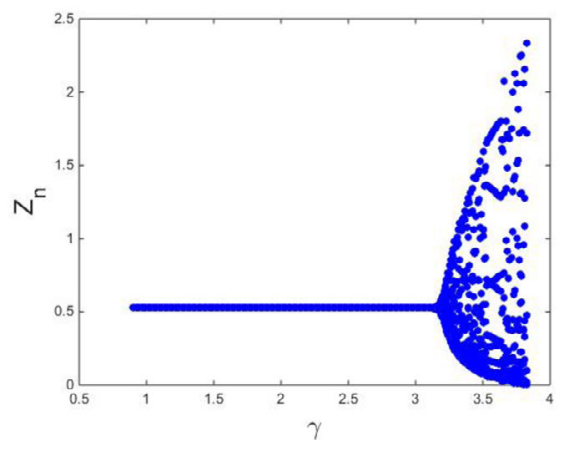

(b)

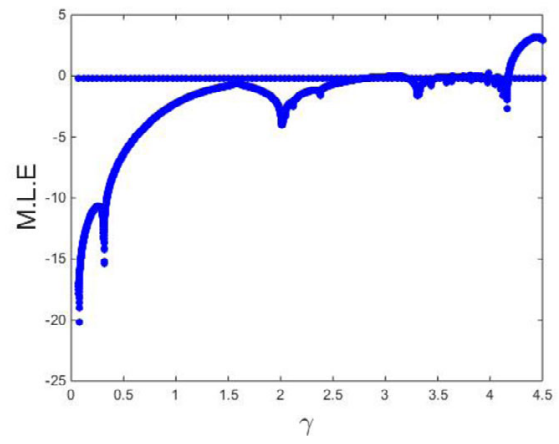

(c)

Figure $33(\mathbf{a})-3(\mathbf{b}) \mathrm{N}-\mathrm{S}$ bifurcation diagrams of phytoplankton-zooplankton model (8) with $\gamma \in[0.9,4.95]$ 3(b) Maximum Lyapunov exponents corresponding to 3(a) $-3(\mathbf{b})$ with $(0.54,0.85)$

tively unstable) node if $0<\gamma<0.12657824922905964$ (respectively $\gamma>$ $0.12657824922905964)$. For this, if $\gamma=0.012<0.12657824922905964$, then from Fig. 4(a), $F_{P Z}^{+}(0.6282650564308631,1.6522521579455332)$ of phytoplankton-zooplankton model (8) is a stable node. In a similar faction, Fig. 4(b)-4(d) also indicates the fact that for the bifurcation value $\gamma=0.0123,0.11,0.112<0.12657824922905964$ of phytoplanktonzooplankton model (8) is a stable node. Further recall that if $\gamma>0.12657824922905964$, then $F_{P Z}^{+}\left(\sqrt{\frac{\omega \nu^{2}}{1-\omega}}, \frac{\beta \nu\left(\sqrt{1-\omega}-\sqrt{\omega \nu^{2}}\right)}{\sqrt{\omega}(1-\omega)}\right)$ becomes unstable, and meanwhile a flip bifurcation occurs, i.e., if $\gamma=0.13>0.12657824922905964$, then by mathematical computation from (83) one gets $\Gamma_{1}=-1.1111111 \neq 0$. Moreover, from (84) one gets $\Gamma_{2}=0.3722876096102278>0$, 


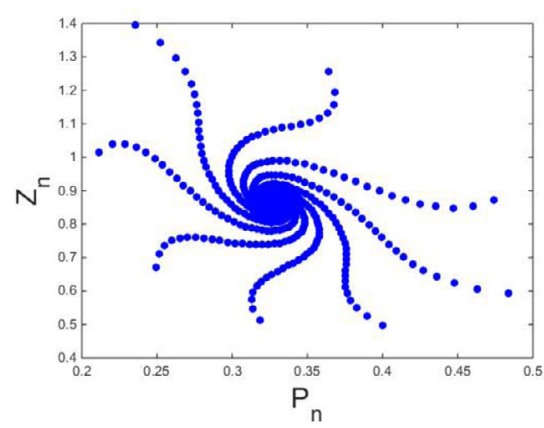

(a) $\gamma=0.012$ with $(0.2484,0.2595)$

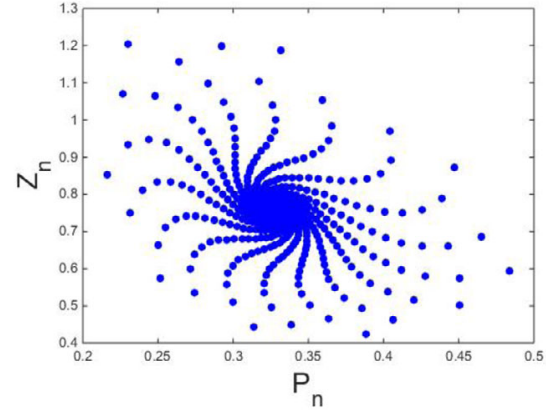

(c) $\gamma=0.11$ with $(0.2484,0.2595)$

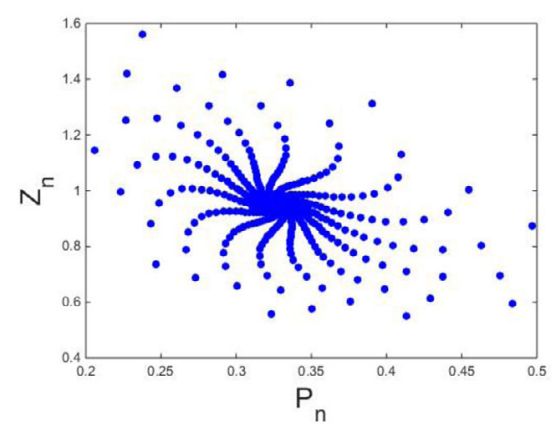

(b) $\gamma=0.0123$ with $(0.2484,0.2595)$

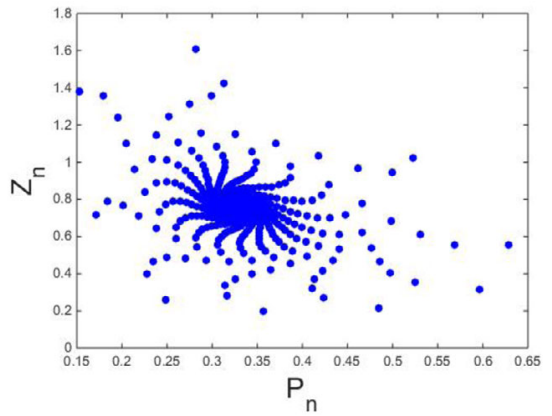

(d) $\gamma=0.112$ with $(0.2484,0.2595)$

Figure 4 Stable node of phytoplankton-zooplankton model (8)

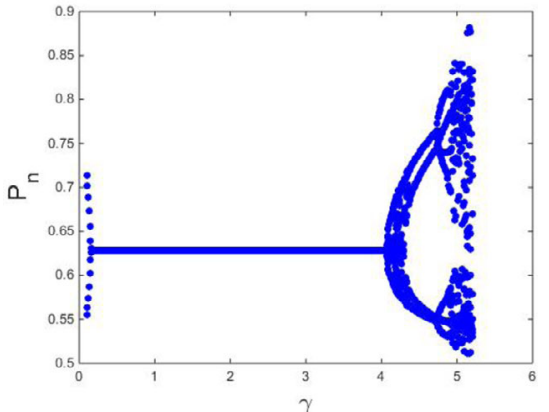

(a)

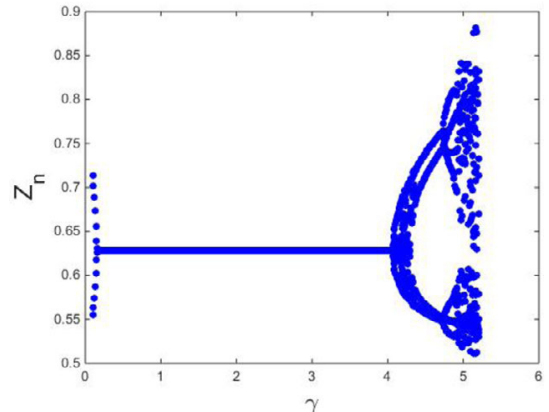

(b)

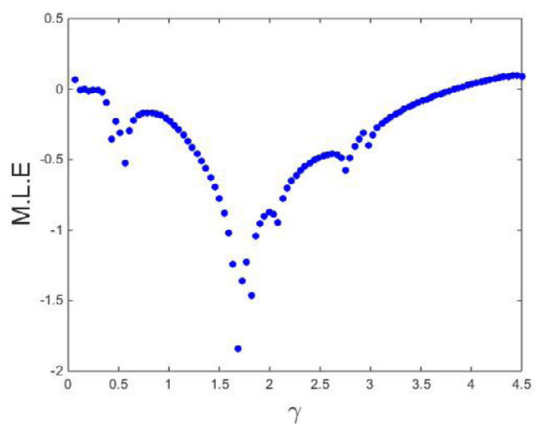

(c)

Figure 5 5(a)-5(b) Flip bifurcation diagrams of phytoplankton-zooplankton model (8) with $\gamma \in[0.1,5.2] 3($ b) Maximum Lyapunov exponents corresponding to $5(\mathbf{a})-5(\mathbf{b})$ with $(0.84,0.75)$ 


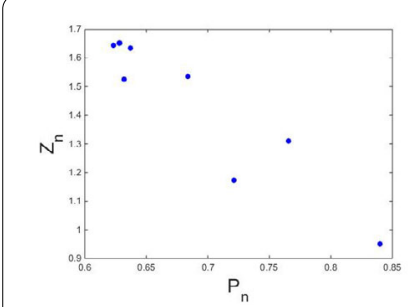

(a) $\gamma=2.1$ with $(0.84,0.95)$

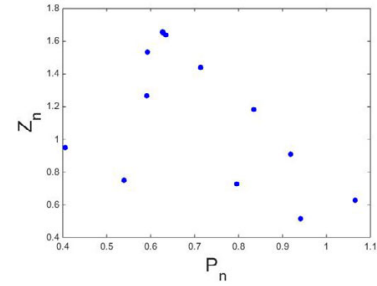

(d) $\gamma=2.73$ with $(0.5432,0.75)$

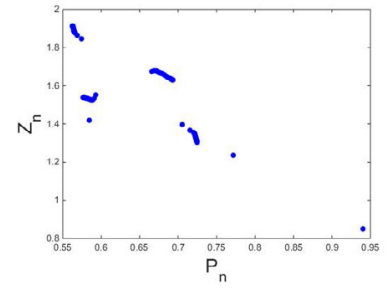

(g) $\gamma=4.4$ with $(0.94,0.85)$

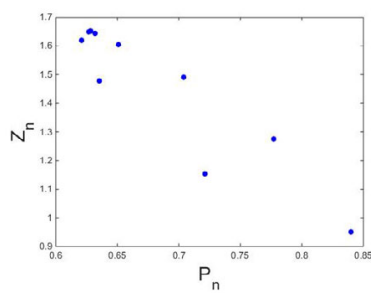

(b) $\gamma=1.9$ with $(0.84,0.95)$

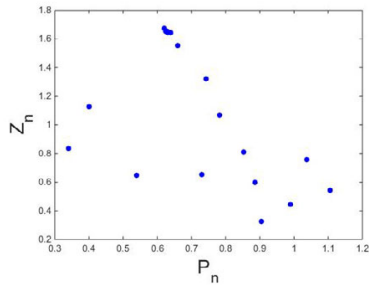

(e) $\gamma=2.7$ with $(0.64,0.85)$

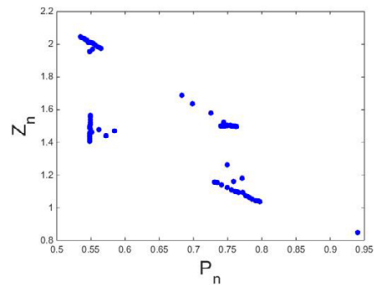

(h) $\gamma=4.8$ with $(0.94,0.85)$

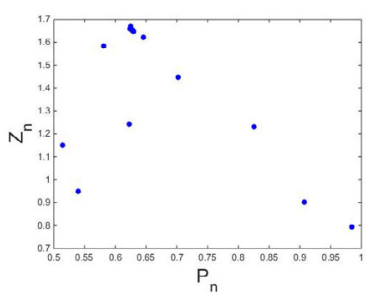

(c) $\gamma=2.7$ with $(0.54,0.95)$

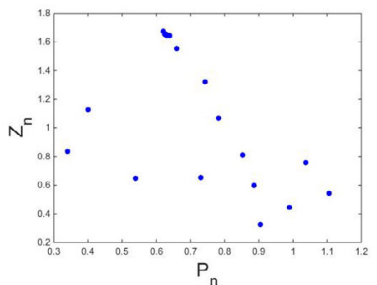

(f) $\gamma=2.7$ with $(0.54,0.65)$

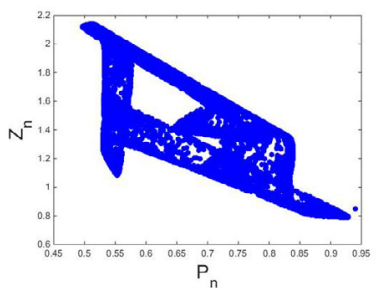

(i) $\gamma=5.2$ with $(0.94,0.85)$

Figure 6 Complex and chaotic behavior of phytoplankton-zooplankton model (8)

which indicates the fact that stable period-2 points bifurcate from $F_{P Z}^{+}\left(\sqrt{\frac{\omega \nu^{2}}{1-\omega}}\right.$, $\frac{\beta v\left(\sqrt{1-\omega}-\sqrt{\omega \nu^{2}}\right)}{\sqrt{\omega}(1-\omega)}$, and hence a flip bifurcation diagram along with maximum Lyapunov exponents is plotted and presented in Fig. 5. Figure 6 has been drawn to show the complex dynamics of phytoplankton-zooplankton model (8) with orbits of period-8, 9, 10, 11, 14, 15, whereas Fig. 6(g)-6(i) shows the chaotic behavior.

Hereafter we prove the validity of the obtained results in Sect. 6. For instance, if $h=0.9$, $\beta=3.7, v=0.6, \omega=0.523$, then from (90), (91), and (92) one gets

$$
\begin{aligned}
& L_{1}: k_{1}-4.3237312072633385 k_{2}=0, \\
& L_{2}: k_{2}+0.4707=0, \\
& L_{3}: 2 k_{1}-4.3237312072633385 k_{2}-1.9648197207411462 .
\end{aligned}
$$

Hence the lines that are presented in (95), (96), and (97) determine the triangular region that gives $\left|\lambda_{1,2}\right|<1$ (see Fig. 7). Finally, $n$ vs $P_{n}$ and $Z_{n}$ have been plotted for controlled system (85) with $k_{1}=1.0358101854205568, k_{2}=-0.47069999999999995$, which shows that unstable trajectories are stabilized (see Fig. 8 ). 
Figure 7 Region of stability where $\left|\lambda_{1,2}\right|<1$

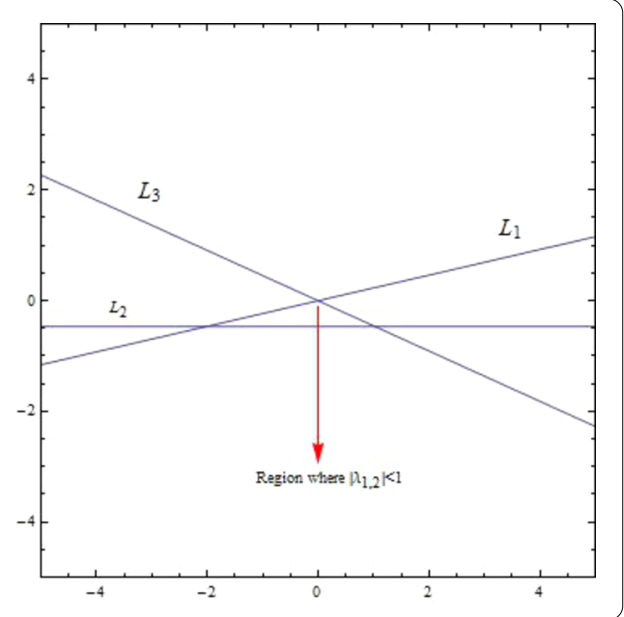

Figure 8 Graphs of $n$ vs $P_{n}$ and $Z_{n}$ for controlled system (85)
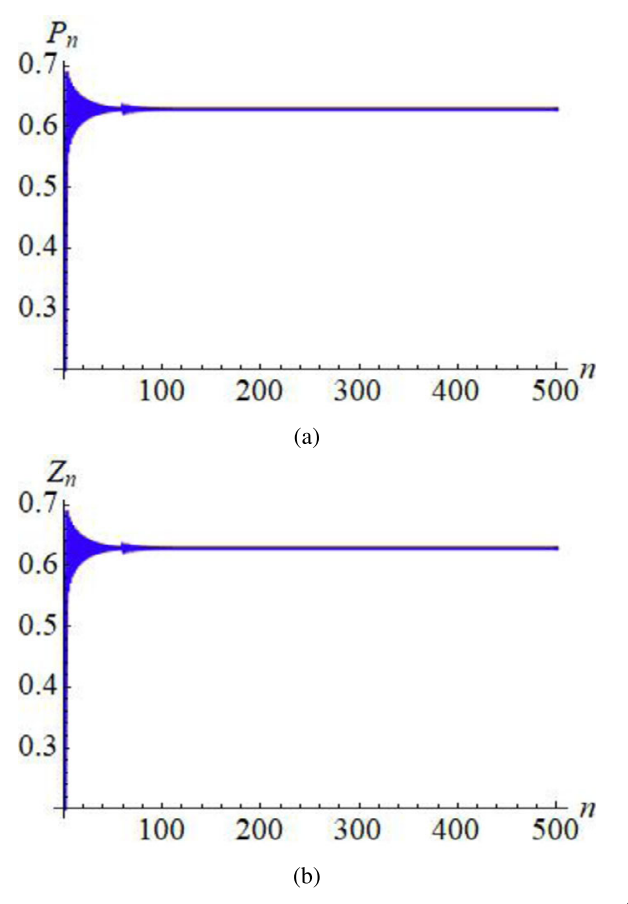

\section{Conclusion}

The work is about the topological classifications around fixed points, periodic points, bifurcations, and chaos control in the discrete-time phytoplankton-zooplankton model (8) in the region $\mathbb{R}_{+}^{2}=\{(P, Z): P, Z \geq 0\}$. We proved that, for all parametric values $\gamma, h, \beta, \omega$, $v$, phytoplankton-zooplankton model (8) has trivial and semitrivial fixed points $F_{00}(0,0)$, $F_{P 0}(1,0)$, but it has an interior fixed point $F_{P Z}^{+}\left(\sqrt{\frac{\omega v^{2}}{1-\omega}}, \frac{\beta \nu\left(\sqrt{1-\omega}-\sqrt{\omega v^{2}}\right)}{\sqrt{\omega}(1-\omega)}\right)$ if $\omega<\min \left\{1, \frac{1}{v^{2}+1}\right\}$. Further we studied the local stability with different topological classifications around each fixed point, and the main findings are presented in Table 2. Next, for phytoplanktonzooplankton model (8), we also studied the existence of periodic points by existing theory. Further we explored the local bifurcations like flip bifurcation, transcription bifurcation, and N-S bifurcation which can be analyzed entirely through changes in the local stabil- 
Table 2 Topological classifications around fixed points of phytoplankton-zooplankton model (8)

\begin{tabular}{|c|c|}
\hline Fixed points & Corresponding behavior \\
\hline$\overline{F_{00}(0,0)}$ & $\begin{array}{l}\text { never sink; source if } \gamma>\frac{2}{h \omega} \text {; saddle if } 0<\gamma<\frac{2}{h \omega} \text {; } \\
\text { non-hyperbolic if } \gamma=\frac{2}{h \omega}\end{array}$ \\
\hline$F_{P 0}(1,0)$ & $\begin{array}{l}\text { sink if } \frac{2\left(v^{2}+1\right)}{\gamma\left(\omega\left(v^{2}+1\right)-1\right)}<h<\frac{2}{\beta} ; \\
\text { source if } \frac{2\left(v^{2}+1\right)}{\gamma\left(\omega\left(v^{2}+1\right)-1\right)}>h>\frac{2}{\beta} ; \\
\text { saddle if } h>\max \left\{\frac{2}{\beta}, \frac{2\left(v^{2}+1\right)}{\gamma\left(\omega\left(v^{2}+1\right)-1\right)}\right\} ; \\
\text { non-hyperbolic if } h=\frac{2}{\beta} \text { or } h=\frac{2\left(v^{2}+1\right)}{\gamma\left(\omega\left(v^{2}+1\right)-1\right)}\end{array}$ \\
\hline$F_{P Z}^{+}\left(\sqrt{\frac{\omega v^{2}}{1-\omega}}, \frac{\beta v\left(\sqrt{1-\omega}-\sqrt{\omega v^{2}}\right)}{\sqrt{\omega}(1-\omega)}\right)$ & $\begin{array}{l}\text { stable focus if } \gamma<\frac{\sqrt{1-\omega}-2 \omega\left(\sqrt{1-\omega}-\sqrt{\omega \nu^{2}}\right)}{2 h \omega(1-\omega)\left(\sqrt{1-\omega}-\sqrt{\omega \nu^{2}}\right)} ; \\
\text { unstable focus if } \gamma>\frac{\sqrt{1-\omega}-2 \omega\left(\sqrt{1-\omega}-\sqrt{\omega \nu^{2}}\right)}{2 h \omega(1-\omega)\left(\sqrt{1-\omega}-\sqrt{\omega \nu^{2}}\right)} ; \\
\text { non-hyperbolic if } \gamma=\frac{\sqrt{1-\omega}-2 \omega\left(\sqrt{1-\omega}-\sqrt{\omega \nu^{2}}\right)}{2 h \omega(1-\omega)\left(\sqrt{1-\omega}-\sqrt{\omega \nu^{2}}\right)} ; \\
\text { stable node if } \gamma<\frac{(h \beta-2) \sqrt{1-\omega}-2 h \beta \omega\left(\sqrt{1-\omega}-\sqrt{\omega \nu^{2}}\right)}{h^{2} \beta \omega(1-\omega)\left(\sqrt{1-\omega}-\sqrt{\omega \nu^{2}}\right)} ; \\
\text { unstable node if } \gamma>\frac{(h \beta-2) \sqrt{1-\omega}-2 h \beta \omega\left(\sqrt{1-\omega}-\sqrt{\omega \nu^{2}}\right)}{h^{2} \beta \omega(1-\omega)\left(\sqrt{1-\omega}-\sqrt{\omega v^{2}}\right)} ; \\
\text { non-hyperbolic if } \gamma=\frac{(h \beta-2) \sqrt{1-\omega}-2 h \beta \omega\left(\sqrt{1-\omega}-\sqrt{\omega \nu^{2}}\right)}{h^{2} \beta \omega(1-\omega)\left(\sqrt{1-\omega}-\sqrt{\omega \nu^{2}}\right)}\end{array}$ \\
\hline
\end{tabular}

ity properties around fixed points $F_{00}(0,0), F_{P 0}(1,0), F_{P Z}^{+}\left(\sqrt{\frac{\omega \nu^{2}}{1-\omega}}, \frac{\beta \nu\left(\sqrt{1-\omega}-\sqrt{\omega \nu^{2}}\right)}{\sqrt{\omega}(1-\omega)}\right)$, periodic orbits, or other invariant sets as parameters crossing through bifurcation value to understand model (8) more deeply. So, it is proved that around a trivial fixed point there exists a transcritical bifurcation if $\left.(\gamma, h, \beta, \omega, \nu) \in \mathcal{T}\right|_{F_{00}(0,0)}=\left\{(\gamma, h, \beta, \omega, \nu), \gamma=\frac{2}{h \omega}\right\}$, but around trivial and semitrivial fixed points there exists no flip bifurcation if $(\gamma, h, \beta, \omega, \nu) \in$ $\left.\mathcal{F}\right|_{F_{00}(0,0)}=\left\{(\gamma, h, \beta, \omega, \nu), \gamma=\frac{2}{h \omega}\right\}$ and $\left.(\gamma, h, \beta, \omega, \nu) \in \mathcal{F}\right|_{F_{P 0}(1,0)}=\left\{(\gamma, h, \beta, \omega, \nu), h=\frac{2}{\beta}\right\}$, respectively. We also investigated that around $F_{P Z}^{+}\left(\sqrt{\frac{\omega v^{2}}{1-\omega}}, \frac{\beta v\left(\sqrt{1-\omega}-\sqrt{\omega v^{2}}\right)}{\sqrt{\omega}(1-\omega)}\right)$, phytoplanktonzooplankton model (8) undergoes both Neimark-Sacker and flip bifurcations if parameters go through the curves $\left.(\gamma, h, \beta, \omega, \nu) \in \mathcal{N}\right|_{F_{P Z}^{+}\left(\sqrt{\frac{\omega \nu^{2}}{1-\omega}}, \frac{\beta \nu\left(\sqrt{1-\omega}-\sqrt{\omega \nu^{2}}\right)}{\sqrt{\omega}(1-\omega)}\right)}=\{(\gamma, h, \beta, \omega, \nu), \gamma=$ $\left.\frac{\sqrt{1-\omega}-2 \omega\left(\sqrt{1-\omega}-\sqrt{\omega \nu^{2}}\right)}{2 h \omega(1-\omega)\left(\sqrt{1-\omega}-\sqrt{\omega \nu^{2}}\right)}\right\}$ and $\left.(\gamma, h, \beta, \omega, \nu) \in \mathcal{F}\right|_{F_{P Z}^{+}\left(\sqrt{\frac{\omega \nu^{2}}{1-\omega}}, \frac{\beta \nu\left(\sqrt{1-\omega}-\sqrt{\omega \nu^{2}}\right)}{\sqrt{\omega}(1-\omega)}\right)}=\{(\gamma, h, \beta, \omega, \nu), \gamma=$ $\left.\frac{h \beta \sqrt{1-\omega}-2 \sqrt{1-\omega}-2 h \beta \omega\left(\sqrt{1-\omega}-\sqrt{\omega \nu^{2}}\right)}{h^{2} \beta \omega(1-\omega)\left(\sqrt{1-\omega}-\sqrt{\omega \nu^{2}}\right)}\right\}$, respectively. From the viewpoint of biology, the occurrence of Neimark-Sacker implies that there exist periodic or quasi-periodic oscillations between phytoplankton and zooplankton populations. Further the state feedback control method is utilized to stabilize chaos existing in the discrete-time phytoplanktonzooplankton model. Finally, the obtained results are verified not only numerically but also by showing the complex dynamics with orbits of period-8, 9, 10, 11, 14, 15 and chaotic behavior of the discrete-time phytoplankton-zooplankton model.

Acknowledgements

This work was supported by the Higher Education Commission of Pakistan.

Funding

Not applicable.

Availability of data and materials

Not applicable. 
Authors' contributions

Both authors have read and approved the final manuscript.

\section{Publisher's Note}

Springer Nature remains neutral with regard to jurisdictional claims in published maps and institutional affiliations.

Received: 30 December 2020 Accepted: 19 July 2021 Published online: 14 September 2021

\section{References}

1. Truscott, J., Brindley, J.: Ocean plankton populations as excitable media. Bull. Math. Biol. 56(5), 981-998 (1994)

2. Chattopadhayay, J., Sarkar, R., Mandal, S.: Toxin-producing plankton may act as a biological control for planktonic blooms - field study and mathematical modelling. J. Theor. Biol. 215(3), 333-344 (2002)

3. Anderson, D.: Toxic algal blooms and red tides: a global perspective. In: Red Tides: Biology, Environmental Science and Toxicology, pp. 11-16. Elsevier, New York (1989)

4. Smayda, T:: Novel and Nuisance Phytoplankton Blooms in the Sea: Evidence for a Global Epidemic. RWS-North Sea Directorate, pp. 29-40 (1990)

5. Hallegraeff, G.: A review of harmful algal blooms and their apparent global increase. Phycologia 32(2), 79-99 (1993)

6. Blaxter, J., Southward, A.: Advances in Marine Biology. Academic Press, London (1997)

7. Stoermer, E., Smol, J.: The Diatoms. Cambridge University Press, Cambridge (1999)

8. Guckenheimer, J., Holmes, P.: Nonlinear Oscillations, Dynamical Systems and Bifurcation of Vector Fields. Springer, New York (1983)

9. Kuznetsov, Y.: Elements of Applied Bifurcation Theory, 3rd edn. Springer, New York (2004)

10. Sohel Rana, S.: Chaotic dynamics and control of discrete ratio-dependent predator-prey system. Discrete Dyn. Nat. Soc. 2017, Article ID 4537450 (2017)

11. Al-Basyouni, K., Khan, A.: Discrete-time predator-prey model with bifurcations and chaos. Math. Probl. Eng. 2020, Article ID 8845926 (2020)

12. Mehrjooee, O., Fathollahi Dehkordi, S., Habibnejad Korayem, M.: Dynamic modeling and extended bifurcation analysis of flexible-link manipulator. Mech. Based Des. Struct. Mach. 48(1), 87-110 (2020)

13. Chakraborty, P., Ghosh, U., Sarkar, S.: Stability and bifurcation analysis of a discrete prey-predator model with square-root functional response and optimal harvesting. J. Biol. Syst. 28(01), 91-110 (2020)

14. Liu, W., Cai, D.: Bifurcation, chaos analysis and control in a discrete-time predator-prey system. Adv. Differ. Equ. 2019(1), $11(2019)$

15. Beddington, J., Free, C., Lawton, J.: Dynamic complexity in predator-prey models framed in difference equations. Nature 225, 58-60 (1975)

16. Chen, F.: Permanence and global attractivity of a discrete multispecies Lotka-Volterra competition predator-prey system. Appl. Math. Comput. 181, 3-12 (2006)

17. Fang, Q., Li, X., Cao, M.: Dynamics of a discrete predator-prey system with Beddington-DeAngelis function response. Appl. Math. 3, 389-394 (2012)

18. Agiza, H., Elabbssy, E.: Chaotic dynamics of a discrete prey-predator model with Holling type II. Nonlinear Anal., Real World Appl. 10, 116-129 (2009)

19. Liu, X., Xiao, D.: Complex dynamic behaviors of a discrete-time predator-prey system. Chaos Solitons Fractals 32 , 80-94 (2007)

20. Khan, A., Ma, J., Xiao, D.: Bifurcations of a two-dimensional discrete time plant-herbivore system. Commun. Nonlinear Sci. Numer. Simul. 2016(39), 185-198 (2016)

21. Khan, A., Ma, J., Xiao, D.: Global dynamics and bifurcation analysis of a host-parasitoid model with strong Allee effect. J. Biol. Dyn. 11(1), 121-146 (2017)

22. Elaydi, S.: An Introduction to Difference Equations. Springer, New York (1996)

23. Lynch, S.: Dynamical Systems with Applications Using Mathematica. Birkhäuser, Boston (2007)

\section{Submit your manuscript to a SpringerOpen ${ }^{\circ}$ journal and benefit from:}

- Convenient online submission

- Rigorous peer review

Open access: articles freely available online

- High visibility within the field

- Retaining the copyright to your article

Submit your next manuscript at $>$ springeropen.com 